\title{
Abelianization of BPS quivers and the refined Higgs index
}

\author{
Seung-Joo Lee, ${ }^{a}$ Zhao-Long Wang ${ }^{a, b}$ and Piljin Yi ${ }^{a}$ \\ ${ }^{a}$ School of Physics, Korea Institute for Advanced Study, \\ Seoul 130-722, Korea \\ ${ }^{b}$ Institute of Modern Physics, Northwest University, \\ Xian 710069, China \\ E-mail: s.lee@kias.re.kr, zlwang@kias.re.kr, piljin@kias.re.kr
}

\begin{abstract}
We count Higgs "phase" BPS states of general non-Abelian quiver, possibly with loops, by mapping the problem to its Abelian, or toric, counterpart and imposing Weyl invariance later. Precise Higgs index computation is particularly important for quivers with superpotentials; the Coulomb "phase" index is recently shown to miss important BPS states, dubbed intrinsic Higgs states or quiver invariants. We demonstrate how the refined Higgs index is naturally decomposed to a sum over partitions of the charge. We conjecture, and show in simple cases, that this decomposition expresses the Higgs index as a sum over a set of partition-induced Abelian quivers of the same total charge but generically of smaller rank. Unlike the previous approach inspired by a similar decomposition of the Coulomb index, our formulae compute the quiver invariants directly, and thus offer a self-complete routine for counting BPS states.
\end{abstract}

Keywords: Black Holes in String Theory, Extended Supersymmetry, D-branes, Differential and Algebraic Geometry

ARXIV EPRINT: 1310.1265 


\section{Contents}

1 Quivers and indices 1

2 How to compute Higgs index 4

2.1 Abelianization and the lift 5

$\begin{array}{ll}2.2 & \text { Indices for quivers without loops }\end{array}$

2.3 An illustration: Grassmannian $X \quad 9$

2.4 Loops, the superpotential, and the normal bundle 10

3 Examples with a loop: triangular quivers 11

$\begin{array}{lll}3.1 & \text { A simplest non-Abelian triangular quiver } & 11\end{array}$

$\begin{array}{ll}3.2 & \text { General triangular quivers } \\ & 3.2 .13\end{array}$

$\begin{array}{ll}3.2 .1 \text { Consistency check: quiver mutation } & 15\end{array}$

$\begin{array}{ll}3.2 .2 & \text { Quiver invariants revisited } \\ \end{array}$

$\begin{array}{lll}4 & \text { Abelianized Higgs index is a partition sum } & 17\end{array}$

$\begin{array}{ll}4.1 \text { Coulomb index as a partition sum } & 17\end{array}$

$\begin{array}{ll}4.2 & \text { Is the Higgs index also a partition sum? } \\ \end{array}$

A Intersection structures of toric varieties $\quad 25$

B The ambient D-term variety and maximal reduced quivers 26

\section{Quivers and indices}

The low energy dynamics of BPS particles or BPS black holes in four dimensions are most succinctly captured by quiver dynamics, which originate from wrapped D-brane picture of such particles [1, 2] compactified on a Calabi-Yau 3-fold, where particle-like BPS states arise from D3-branes wrapped on special Lagrangian 3-cycles. When the 3-cycle has topology of $S^{3}$, the low energy dynamics of $n$ wrapped D3-branes would be $\mathrm{U}(\mathrm{n})$ gauged quantum mechanics with four supercharges. In the phase where the symmetry is broken to $\mathrm{U}(1)^{\mathrm{n}}$, the triplet eigenvalues of the Cartan vector multiplets encode the position of $n$ BPS particles along the noncompact $\mathbb{R}^{3}$, while the residual Weyl group shuffles these $n$ identical particles. When more than one 3-cycles are involved, each wrapped by D3-branes as well, we find additional chiral multiplets, in bi-fundamentals, arising from open strings between each pair of D3's. The number of such chiral fields is identified with the intersection number.

The quiver dynamics itself can be further approximated by integrating out either vector multiplets or chiral multiplets. The two such descriptions are called Higgs and Coulomb "phase" descriptions, respectively. The word "phase" here is very misleading, although it 
is used conventionally, as the quiver dynamics in question is an one-dimensional system and thus the vacuum expectation values do not imply superselection sectors. It merely refers to particular integrating out procedure, which may or may not be reliable depending on the massgap, although Supersymmetry tends to protect quantities like index further. When both sides are reliable, we expect the computed indices from the two sides to agree with each other. This is the case, as far as we know, when the quiver has no loop [1,3].

Generally speaking, the Higgs description, better suited for large Fayet-Iliopoulos (FI) constants, $\theta$ 's, is more reliable as the massive vector multiplet fields being integrated out tend to have uniformly large mass of order $m^{2} \sim \theta$, justifying the procedure. The Coulomb description, suitable for small $\theta$ 's, provides a more intuitive picture of the wall-crossing via its multi-center picture; the positions of charge centers are encoded in the Cartan part of the vector multiplets. While physically more appealing, this latter Coulomb description turns out to involve various subtleties. Identification of the correct index theorem was rigorously argued only very recently [4], and does not follow from naive truncation to classically flat part of collective coordinates; one must really invoke a localization procedure as some of the classical massgaps are no larger than the quantum mechanical massgaps associated with the finite volume of the classical moduli space [4]. The massgap issue becomes more serious in the presence of the so-called scaling regime, where one finds classical multi-center solutions with mutual distances arbitrarily small. For the latter class, for which the relevant quiver dynamics must have at least one closed loop, the naive massgap $m \sim 1 / \theta$ for the chiral multiplets fails completely, with no obvious localization procedure available.

Nevertheless, in the absence of scaling regimes (thus, in the absence of loop), the Coulomb description is very useful as it can be derived as the BPS soliton or black hole dynamics from the underlying $N=2 D=4$ theory, and it shows a clear intuitive picture of wall-crossing phenomena as multi-particle bound state physics [5-10]. This Coulomb description has been derived, ab initio, for Seiberg-Witten dyons [4, 11], i.e., from the field theory itself as low energy dynamics of UV-incomplete solitons; as long as we can ensure the individual constituent particles are actually present in the spectrum, the low energy interaction among them are reliable when we stay very close to the relevant marginal stability wall. Regardless of how we view such multi-particle dynamics, a rather complete derivation of the Coulomb index had emerged very recently [4, 12, 13], which was then shown [3] to be equivalent among themselves and to the Kontsevich-Soibelman conjecture [14]. We will briefly revisit this Coulomb index in section 4 .

The natural index in $N=2 D=4$ field theory is the second helicity trace

$$
\Omega(\gamma)=-\frac{1}{2} \operatorname{tr}_{\gamma}(-1)^{2 J_{3}}\left(2 J_{3}\right)^{2}
$$

where trace is over the one-particle Hilbert space of the given charge $\gamma$, and $J_{3}$ is the helicity operator. For four-dimensional $N=2$ field theory, there is a natural equivariant extension, called Protected Spin Character (PSC) [15]

$$
\Omega(\gamma ; y)=-\frac{1}{2} \operatorname{tr}_{\gamma}(-1)^{2 J_{3}}\left(2 J_{3}\right)^{2} y^{2 J_{3}+2 I_{3}}
$$

with $I_{3}$ belonging to $\mathrm{SU}(2)_{\mathrm{R}}$ symmetry. When we factor out the universal halfhypermultiplet factor in the BPS supermultiplets, these reduce to the more familiar Witten- 
type indices as

$$
\Omega(\gamma)=\operatorname{tr}_{\gamma}^{\prime}(-1)^{2 J_{3}}
$$

and

$$
\Omega(\gamma ; y)=\operatorname{tr}_{\gamma}^{\prime}(-1)^{2 J_{3}} y^{2 J_{3}+2 I_{3}} .
$$

In the low energy description of these BPS objects, we effectively compute the latter, after removing the free center-of-mass part of the low energy dynamics.

In particular, for quiver dynamics, PSC descends to [16, 17]

$$
\Omega(\gamma ; y)=\operatorname{tr}_{\gamma}^{\prime}(-1)^{2 J_{3}} y^{2 J_{3}+2 I}
$$

where, as the quiver dynamics is a gauged quantum mechanics with four supercharges, $\mathrm{SU}(2)_{\mathrm{J}}$ rotation generated by $J_{a}$ 's is now an R-symmetry of the quiver dynamics while $I$ generates the other R-symmetry U(1) I. For Higgs "phase," it has been argued that this equivariant index is computed by (shifted) Hirzebruch characters,

$$
\Omega_{\mathrm{Higgs}}(y)=\sum_{p=0}^{d} \sum_{q=0}^{d}(-1)^{p+q-d} y^{2 p-d} \operatorname{dim} H^{(p, q)},
$$

where $d$ is the complex dimension of the Higgs moduli space of the quiver. The Higgs moduli space is always Kähler, allowing us to use Hodge decomposition. This collapses to, when $y=1$,

$$
\Omega_{\mathrm{Higgs}}=\sum_{n=0}^{2 d}(-1)^{n-d} \operatorname{dim} H^{(n)},
$$

which is the Euler number times $(-1)^{d}$. In terms of the R-charges of the quiver, $2 J_{3} \rightarrow$ $(p+q)-d=n-d$ and $2 I \rightarrow p-q$.

Actual computation of $\Omega_{\mathrm{Higgs}}$ is available for some subfamilies of quivers. Reineke has given general formulae for the Poincare polynomial of general quivers without loops [18]; this can be thought of as Higgs counterpart of the Coulomb index computations mentioned above. More interesting are $\Omega_{\text {Higgs }}$ for quivers with loops, which neither of the above can address. The equivariant index of an arbitrary Abelian cyclic quiver with generic superpotential was computed in refs. [16], and along the way was found a new class of BPS states [16, 17], called intrinsic Higgs states. They were found to be wall-crossing-safe, invisible from the Coulomb description, and of zero angular momenta. They are typically far more numerous than Coulomb "phase" states, given a quiver with loops; these states are clearly important ingredients in understanding microstates of $N=2$ single-center black holes, but they also appear in some field theory BPS spectra, such as that of $N=2^{*} \mathrm{SU}(2)$ theory [19].

A challenge we wish to face in this note is how to generalize these Higgs index computations to general non-Abelian quivers with superpotentials. For Abelian quivers that have been studied, the index is computable relatively easily because Higgs moduli spaces are embedded in toric varieties. For non-Abelian cases, one encounters more general symplectic quotients by non-Abelian groups and, with superpotentials, has to intersect the zero 
loci of sections of vector bundles over such varieties. A general procedure that can recast computation of indices on such spaces to a problem in a bigger toric variety is known in the mathematical literature [20-22], which we will adapt to the problem at hand. This effectively replaces any given non-Abelian quiver by an Abelian one with the same total charge and of the same rank, by splitting each non-Abelian node, say of rank $n$, to $n$ Abelian nodes. Section 2 will declare the procedure and section 3 will elaborate with examples.

An interesting corollary of this Abelianization method is that the end results have some similarity to the Coulomb "phase" wall-crossing formulae in refs. [4, 12]. In the latter, the gauge symmetry is spontaneously broken to the Cartan part, with massless bosons encoding the positions of the particles. The non-Abelian nature of the quiver enters only at the last step, via the Weyl projection, which has been shown to result in a sum over partitions of the charge [4]. Our Higgs "phase" computation of index is very similar in spirit in that we rely on Cartan subalgebra and the Weyl projection in the end. This naturally leads us to suspect that our Abelianization procedure parallels in some sense the index computation on the Coulomb side. In section 4, we elaborate this idea further for simple examples, and offer a conjecture on how the Higgs index can also be naturally written as a sum over partitions of the charge in a manner that parallels the Coulomb index partition sum.

After this work was completed, ref. [36] appeared in the arXiv. There an intriguing transformation rule is suggested for the quiver invariants between different (non-Abelian) quivers related by mutation. Our formulae provided in this note should be capable of verifying explicitly non-Abelian examples in their work.

\section{How to compute Higgs index}

As already explained in the previous section, Higgs phase index can be computed as the Euler number $\chi(M)$ of the Higgs moduli space $M$, which, as we will shortly see, is constructed as a complete intersection via F-terms, embedded in the D-term variety $X$. As is well-known, with the aid of adjunction formula, certain invariants of $M$, including its Euler number $\chi(M)$, are expressible in terms of the ambient space data [23]. In case of Abelian quivers, the corresponding ambient space $X$ is a toric variety and hence, one can easily extract relevant invariants in a straight-forward manner, by using simple combinatorial prescriptions from toric geometry. On the other hand, for general quivers with non-Abelian nodes, it is more difficult to deal with the resulting D-term variety.

The upshot of the computational prescription for Higgs phase index is to first "Abelianize" the quiver and to make use of the corresponding "toric" quiver variety $\tilde{X}$ as well as a complete intersection $\tilde{M}$ therein. One can then apply the usual toric techniques. In this section, we shall briefly describe the index prescription in full generality at the risk of making the presentation abstract; some concrete, illustrative examples will follow in the ensuing section for triangular quivers.

Before we proceed, it is important to note that we work in individual branches of the quiver. In other words, we presume a definite choice of FI parameters $\theta$. For each given branch, the Higgs vacuum moduli space can be obtained via two steps; first, we perform a symplectic reduction using D-term conditions, then, if a loop is present, further impose 
F-term conditions. However, as was seen in ref. [17] for Abelian quivers, we can make life slightly easier by noticing that F-terms tend to simplify things. To make the long story short, having a nontrivial F-term subvariety inside the D-term variety often demands that some bi-fundamentals associated with certain pairs of nodes should be set to zero. This is done to reduce the number of F-terms, because F-terms tend to kill entire Higgs moduli space. For each branch of the quiver, we end up freezing certain sets of bi-fundamental fields to zero in order to obtain a nontrivial $M$, which "reduces" the quiver to be without loops by removing links. See section 2 of ref. [17] or appendix B here for an elaboration on this phenomenon.

\subsection{Abelianization and the lift}

Given a quiver $\mathcal{Q}$ with the gauge group $G=\prod_{v=1}^{N} \mathrm{U}\left(\mathrm{r}_{\mathrm{v}}\right)$ and given a choice of branch (or choice of FI constants), we have $X=\mu_{G}^{-1}(0) / G$ where $\mu_{G}$ is the moment map from the D-term, with the shift by FI constants $\theta$ understood. Then we obtain the true moduli space $M$ by further imposing F-term conditions. The gauge group that actually participates in the quotient is $G / \mathrm{U}(1)$ since there is always one overall $\mathrm{U}(1)$ that acts trivially on all chiral fields. Again, the choice of branch imposes on us to set certain bi-fundamental fields to zero identically, for otherwise $M$ is empty.

To such a non-Abelian quiver $\mathcal{Q}$, we associate an Abelianized quiver $\tilde{\mathcal{Q}}$, obtained by splitting each of the non-Abelian nodes of $\mathcal{Q}$, say, of rank $r_{v}$, into $r_{v}$ Abelian nodes, and simply duplicating the arrows as well as the FI constants. See figures 2 and 3 for an example. Via this Abelianization, we reduce the gauge multiplets to those associated with the Cartan subgroup $T=\prod_{v=1}^{N} \mathrm{U}(1)^{\mathrm{r}_{\mathrm{v}}}$, but keep the same bi-fundamental field contents. (Again, $T / \mathrm{U}(1)$ acts nontrivially on the chiral fields.) With such an Abelianized quiver $\tilde{\mathcal{Q}}$, we end up in the territory of toric geometry. Keeping the same FI parameters, we find the D-term induced variety, $\tilde{X}=\mu_{T}^{-1}(0) / T$, and the subvariety $\tilde{M}$ obtained by imposing F-term conditions as well. Thus, $\tilde{M}$ can be thought of as the Higgs moduli space of $\tilde{\mathcal{Q}}$ in the given branch. Finally, a useful intermediary that will eventually connect the two D-term varieties $X$ and $\tilde{X}$ is the space

$$
Y:=\mu_{G}^{-1}(0) / T
$$

which can be regarded as a bundle over $X$ and also a subvariety of $\tilde{X}$.

Refs. [22, 24] lay down a simple procedure for lifting topological invariants on $X$ to $\tilde{X}$, thereby bridging the two spaces. For any given cohomology class $a \in H^{\star}(X)$, the bridging rule states that

$$
\int_{X} a=\frac{1}{|W|} \int_{\tilde{X}} \hat{a} \wedge e(\boldsymbol{\Delta})
$$

where $W$ is the Weyl group of the gauge group $G$ for the non-Abelian quiver, and $e(\boldsymbol{\Delta})$ is the Euler class of $\boldsymbol{\Delta}$, the Whitney sum of line bundles associated with the "off-diagonal" part, $G / T$, of the gauge group, that is,

$$
\Delta \equiv \bigoplus_{\alpha \in \Delta} \mathcal{L}_{\alpha} .
$$




$$
\begin{gathered}
Y=\mu_{G}^{-1}(0) / T \longrightarrow \tilde{X}=\mu_{T}^{-1}(0) / T \\
\downarrow \\
\downarrow \\
M \stackrel{\iota}{F=0} X=\mu_{G}^{-1}(0) / G
\end{gathered}
$$

Figure 1. Schematics of the Abelianization of the index computation. The main point is that an index computation on $X$, or on its submanifold $M$, can be lifted to a far more straightforward computation in the toric variety $\tilde{X}$, obtained as the symplectic reduction by the Cartan subgroup $T$ of the gauge group $G$. $\mu_{H}$ is the symplectic moment map associated with the group $H$ acting on the flat Kähler space of chiral multiplet scalars.

Note that $\Delta$ denotes the set of roots of $G$ while $\Delta$ in bold denotes the corresponding vector bundle. This bundle is naturally decomposed as $\boldsymbol{\Delta}=\boldsymbol{\Delta}_{+} \oplus \boldsymbol{\Delta}_{-}$according to the usual decomposition of $\boldsymbol{\Delta}$ into the positive and the negative parts. Here, $\boldsymbol{\Delta}_{+}$is the (holomorphic) vector bundle that is tangent to the fibre of $\pi: Y \rightarrow X$. We will shortly see how to express $e(\boldsymbol{\Delta})$ in terms of the toric data for $\tilde{X}$.

The nontrivial part of the bridging rule (2.1) is obviously the lift of $a \in H^{\star}(X)$, denoted by $\hat{a} \in H^{\star}(\tilde{X})$. Lift $\hat{a}$ is defined via the intermediary, $Y=\mu_{G}^{-1}(0) / T$, which naturally admits an inclusion $\iota: Y \hookrightarrow \tilde{X}$ and a projection $\pi: Y \rightarrow X$, in such a way that the relation

$$
\pi^{\star} a=\iota^{\star} \hat{a}
$$

holds on $Y$. While this does not determine the lift $\hat{a}$ uniquely, given $a$, whatever ambiguity there might be is killed by $e(\boldsymbol{\Delta})$ that follows on the right hand side of (2.1). When the cohomology element $a$ is a multiplicative class $m$ associated with the (holomorphic) tangent bundle $\mathcal{T} X$, its lift turns out to be given as [24]

$$
\widehat{m(\mathcal{T} X})=\frac{m(\mathcal{T} \tilde{X})}{m\left(\boldsymbol{\Delta}_{+}\right) \wedge m\left(\boldsymbol{\Delta}_{-}\right)}=\frac{m(\mathcal{T} \tilde{X})}{m(\boldsymbol{\Delta})}
$$

In the next section, we will see how this arises for general quiver varieties in the course of evaluating index by directly constructing a lifted bundle $\widehat{\mathcal{T} X}$ over $\tilde{X}$ such that $\pi^{\star} \mathcal{T} X=$ $\iota^{\star} \widehat{\mathcal{T} X}$.

\subsection{Indices for quivers without loops}

For quivers without loops, and thus, with no F-terms present, the above prescription applies directly and simply since the Higgs moduli space is a symplectic reduction, $X$, of the flat space of bi-fundamental chiral fields. When the quiver has a loop, the superpotential will complicate the space further via F-term constraints, which we will address in subsection 2.4. 
The simplest invariant is the Euler number of $X$,

$$
\chi(X)=\sum_{n}(-1)^{n} b_{n}(X),
$$

that counts the Higgs BPS states when the quiver in question has no loops and thus no F-terms. In terms of the Chern class, $c$, we find [24]

$$
\begin{aligned}
\Omega[X] & =(-1)^{d} \chi(X) \\
& =(-1)^{d} \int_{X} c(\mathcal{T} X) \\
& =\frac{(-1)^{d}}{|W|} \int_{\tilde{X}} \widehat{c(\mathcal{T} X)} \wedge e(\boldsymbol{\Delta}) \\
& =\frac{(-1)^{d}}{|W|} \int_{\tilde{X}} c(\mathcal{T} \tilde{X}) \wedge \frac{e(\boldsymbol{\Delta})}{c(\boldsymbol{\Delta})},
\end{aligned}
$$

where $d \equiv \operatorname{dim}_{\mathbb{C}}(X)$ is the complex dimension of the Higgs moduli space. The extra sign factor in front is there so as to count each hypermultiplet as +1 .

A well-known equivariant version of the Euler number is the refined Euler character, available upon the Hodge decomposition as

$$
\chi_{\xi}=\sum_{p \geq 0} \chi^{p} \xi^{p}, \quad \text { with } \quad \chi^{p}=\sum_{q \geq 0}(-1)^{q} h^{p, q},
$$

which reduces to the Euler number when $\xi=-1$. Recall that $\chi_{\xi}(X)$ is computed via the class (see for instance ref. [25])

$$
\operatorname{Td}(\mathcal{T} X) \wedge \operatorname{ch}_{\xi}\left(\mathcal{T}^{*} X\right)
$$

where $\mathrm{Td}$ and $\mathrm{ch}_{\xi}$ are the multiplicative classes associated, respectively, with $f_{\mathrm{Td}}(x)=$ $x /\left(1-e^{-x}\right)$ and $f_{\operatorname{ch}_{\xi}}(x)=1+\xi e^{x}$. The Abelianization asserts that this quantity can be computed as

$$
\chi_{\xi}(X)=\int_{X} \operatorname{Td}(\mathcal{T} X) \wedge \operatorname{ch}_{\xi}\left(\mathcal{T}^{*} X\right)=\frac{1}{|W|} \int_{\tilde{X}} \frac{\operatorname{Td}(\mathcal{T} \tilde{X}) \wedge \operatorname{ch}_{\xi}\left(\mathcal{T}^{*} \tilde{X}\right)}{\operatorname{Td}(\boldsymbol{\Delta}) \wedge \operatorname{ch}_{\xi}\left(\boldsymbol{\Delta}^{*}\right)} \wedge e(\boldsymbol{\Delta}) .
$$

We will show examples of these equivariant and non-equivariant indices in the next section.

Alternatively, we can directly consider the topological class associated with the refined Higgs index

$$
\Omega(y)=(-y)^{-d} \chi_{\xi=-y^{2}},
$$

which will be more useful in interpreting the Abelianization physically in section 4 . For this, it is convenient to view the factor $(-y)^{-d}$ as a (trivial) multiplicative class associated with the constant function $f_{\mathrm{c}}(x)=(-y)^{-1}$, whereby we find $\Omega(y)$ is directly computed by another multiplicative class $\omega_{y}$ associated with the function

$$
\begin{aligned}
f(x) & =f_{\mathrm{c}}(x) \cdot f_{\mathrm{Td}}(x) \cdot f_{\mathrm{ch}_{\xi}}(-x) \\
& =\frac{x}{\left(1-e^{-x}\right)} \cdot\left(y e^{-x}-y^{-1}\right),
\end{aligned}
$$


where $\xi$ has been replaced by $-y^{2}$. In other words,

$$
\Omega(y)[X]=\int_{X} \omega_{y}(\mathcal{T} X), \quad \omega_{y}(\mathcal{T} X) \equiv \prod_{\mu}\left[x_{\mu} \cdot\left(\frac{y e^{-x_{\mu}}-y^{-1}}{1-e^{-x_{\mu}}}\right)\right]
$$

with eigen-forms $x_{\mu}$ of the curvature of the holomorphic tangent bundle $\mathcal{T} X$. Again, we have

$$
\Omega(y)[X]=\frac{1}{|W|} \int_{\tilde{X}} \omega_{y}(\mathcal{T} \tilde{X}) \wedge \frac{e(\boldsymbol{\Delta})}{\omega_{y}(\boldsymbol{\Delta})}
$$

in the lifted form.

To understand the origin of the contribution from $\Delta$ in eq. (2.4) (and consequently in eqs. (2.5), (2.8) and (2.13)), it is useful to consider how the lift $\widehat{\mathcal{T X}}$ of the tangent bundle $\mathcal{T} X$ is related to the tangent bundle $\mathcal{T} \tilde{X}$ of the Abelianized variety $\tilde{X}$. The relevant exact sequence for general quiver can be written as

$$
0 \rightarrow\left[\bigoplus_{i=1}^{r} \mathcal{O}_{i}\right] \bigoplus\left[\bigoplus_{\alpha \in \Delta} \mathcal{L}_{\alpha}\right] \rightarrow \bigoplus_{\rho \in \Sigma^{(1)}} \mathcal{L}_{\rho} \rightarrow \widehat{\mathcal{T} X} \rightarrow 0
$$

with $r=\operatorname{rk} G-1$, where $\mathcal{O}_{i}$ 's are $r$ copies of the trivial line bundle, call it $\mathcal{O}$, over $\tilde{X}$. The label $i$ serves as a reminder how the corresponding Cartan generator determines the map $\mathcal{O}_{i} \rightarrow \bigoplus \mathcal{L}_{\rho}$. In turn, the latter is a sum of line bundles, $\mathcal{L}_{\rho}$, where $\rho$ belongs to the collection of one-dimensional cones, $\Sigma^{(1)}$, in the "fan," $\Sigma$, for the Abelianized toric variety $\tilde{X}$.

For any multiplicative class $m$, then, we have

$$
\widehat{m(\mathcal{T} X})=m(\widehat{\mathcal{T} X})=\frac{\prod_{\rho \in \Sigma^{(1)}} m\left(\mathcal{L}_{\rho}\right)}{[m(\mathcal{O})]^{r} \wedge \prod_{\alpha \in \Delta} m\left(\mathcal{L}_{\alpha}\right)}=\frac{\prod_{\rho \in \Sigma^{(1)}} m\left(\mathcal{L}_{\rho}\right)}{[m(\mathcal{O})]^{r} \wedge m(\boldsymbol{\Delta})}
$$

As the Euler sequence for the Abelianized D-term variety $\tilde{X}$ is given by

$$
0 \rightarrow\left[\bigoplus_{i=1}^{r} \mathcal{O}_{i}\right] \rightarrow \bigoplus_{\rho \in \Sigma^{(1)}} \mathcal{L}_{\rho} \rightarrow \mathcal{T} \tilde{X} \rightarrow 0
$$

we conclude that [24]

$$
\widehat{m(\mathcal{T} X)}=\frac{m(\mathcal{T} \tilde{X})}{m(\boldsymbol{\Delta})}, \quad m(\mathcal{T} \tilde{X})=\frac{\prod_{\rho \in \Sigma^{(1)}} m\left(\mathcal{L}_{\rho}\right)}{[m(\mathcal{O})]^{r}}
$$

Given the toric data for $\tilde{\mathcal{Q}}$, this carries all the information required to express the (equivariant) index as the integral of a specific cohomology class over $\tilde{X}$.

One must determine the intersection structures on $\tilde{X}$, to evaluate the expressions we obtained. One must delve into details of toric geometry technique for this, which we defer to a short appendix and the references therein. 


\subsection{An illustration: Grassmannian $X$}

As an illustration, let us consider the quiver with gauge group $G=\mathrm{U}(\mathrm{r}) \times \mathrm{U}(1)$, the two nodes for which are linked by $\kappa$ arrows. The Higgs moduli space $X=G r(r, \kappa)$ is the Grassmannian, whose indices are of course well-known already. Nevertheless, let us proceed to compute its topological invariants following the Abelianization procedure. The Abelianized variety $\tilde{X}=\left(\mathbb{P}^{\kappa-1}\right)^{r}$ consists of $r$ copies of projective spaces and we denote by $J_{i=1, \ldots, r}$ the Kähler class of each copy. The intersection structure is simple;

$$
\int_{\tilde{X}}\left(J_{1}\right)^{\kappa-1} \wedge\left(J_{2}\right)^{\kappa-1} \wedge \cdots \wedge\left(J_{r}\right)^{\kappa-1}=1
$$

is the only nonvanishing intersection number.

From the Euler sequence (2.16) for $\tilde{X}$, or that for each of the projective spaces

$$
0 \rightarrow \mathcal{O}_{\mathbb{P}^{\kappa-1}} \rightarrow \mathcal{O}_{\mathbb{P}^{\kappa-1}}(1)^{\oplus \kappa} \rightarrow \mathcal{T} \mathbb{P}^{\kappa-1} \rightarrow 0
$$

we find

$$
\begin{aligned}
c(\mathcal{T} \tilde{X}) & =\prod_{i}\left(1+J_{i}\right)^{\kappa}, \\
\operatorname{Td}(\mathcal{T} \tilde{X}) & =\prod_{i}\left(\frac{J_{i}}{1-e^{-J_{i}}}\right)^{\kappa}, \\
\operatorname{ch}_{\xi}\left(\mathcal{T}^{*} \tilde{X}\right) & =\prod_{i} \frac{\left(1+\xi e^{-J_{i}}\right)^{\kappa}}{(1+\xi)}, \\
\omega_{y}(\mathcal{T} \tilde{X}) & =\prod_{i}\left[\left(\frac{J_{i}}{1-e^{-J_{i}}}\right)^{\kappa} \cdot \frac{\left(y e^{-J_{i}}-y^{-1}\right)^{\kappa}}{y-y^{-1}}\right],
\end{aligned}
$$

where the products run over the range $1 \leq i \leq r$. The factors associated with $\Delta$ can be read off from the off-diagonal parts of $\mathrm{U}(\mathrm{r})=\mathrm{G} / \mathrm{U}(1)$. The $J_{i}$ 's are associated with $\mathrm{U}(1)^{\mathrm{r}}=\mathrm{T} / \mathrm{U}(1)$, under which the off-diagonal parts are labeled by a pair of ordered indices, $i \neq j$, which have the charge of the form

$$
(0, \cdots, 0,1,0, \cdots, 0,-1,0, \cdots, 0) .
$$

So, $c_{1}\left(\mathcal{L}_{\alpha=(i j)}\right)=J_{i}-J_{j}$, which leads to

$$
\begin{aligned}
c(\boldsymbol{\Delta}) & =\prod_{i \neq j}\left(1+J_{i}-J_{j}\right), \\
\operatorname{Td}(\boldsymbol{\Delta}) & =\prod_{i \neq j} \frac{J_{i}-J_{j}}{1-e^{-J_{i}+J_{j}}}, \\
\operatorname{ch}_{\xi}\left(\boldsymbol{\Delta}^{*}\right) & =\prod_{i \neq j}\left(1+\xi e^{-J_{i}+J_{j}}\right), \\
\omega_{y}(\boldsymbol{\Delta}) & =\prod_{i \neq j}\left[\left(J_{i}-J_{j}\right) \cdot\left(\frac{y e^{-J_{i}+J_{j}}-y^{-1}}{1-e^{-J_{i}+J_{j}}}\right)\right],
\end{aligned}
$$


and

$$
e(\boldsymbol{\Delta})=\prod_{i \neq j}\left(J_{i}-J_{j}\right) .
$$

These combined, eq. (2.5) (and eq. (2.13), respectively) reproduces the (refined) Higgs index of the Grassmannian faithfully. We will come back to this example in section 4, and try to give the resulting index formula a little more physical interpretation.

\subsection{Loops, the superpotential, and the normal bundle}

Computation of any multiplicative class for $M$ embedded in $X$ is straightforward as long as we understand the normal bundle $\mathcal{N}$ of this embedding. The general rule states that

$$
\int_{M} m(\mathcal{T} M)=\int_{X} m(\mathcal{T} X) \wedge \frac{e(\mathcal{N})}{m(\mathcal{N})} .
$$

For the problem at hand, we are interested in $m=c$ for the unrefined index, and in either $m=\mathrm{Td} \wedge \operatorname{ch}_{\xi}^{*}$ or $m=\omega_{y}$ for the refined one. ${ }^{1}$ For Abelian quivers this general formula has been used very fruitfully in refs. [16, 17, 30], where a new class of BPS states, intrinsic Higgs states, was discovered. For non-Abelian quivers, this is again lifted to the Abelianized form,

$$
\int_{M} m(\mathcal{T} M)=\frac{1}{|W|} \int_{\tilde{X}} m(\mathcal{T} \tilde{X}) \wedge \frac{\widehat{e(\mathcal{N})}}{\widehat{m(\mathcal{N})}} \wedge \frac{e(\boldsymbol{\Delta})}{m(\boldsymbol{\Delta})},
$$

so it remains to understand how the normal bundle $\mathcal{N}$ of $M$ in $X$ is lifted to a bundle $\widehat{\mathcal{N}}$ over $\tilde{M}$ in $\tilde{X}$.

For quivers with a loop, $\mathcal{Q}$, the superpotential $\mathcal{W}$ generates a F-term constraint

$$
\partial \mathcal{W}=0
$$

for each chiral multiplet in the quiver and defines the embedding of $M$ in $X$. Note that, with a generic choice of superpotential and a generic choice of FI constants, the D-term and the F-term constraints are independent. When we Abelianize $\mathcal{Q}$ to $\tilde{\mathcal{Q}}$, we are removing non-Cartan part of the D-term constraints from the data but leave the chiral field contents and the superpotential thereof intact. This shows that, generically the fibre of $\mathcal{N}$ coincides with that of $\tilde{\mathcal{N}}$, i.e., the normal bundle of the Abelianized Higgs moduli space $\tilde{M}$ embedded into its D-term ambient $\tilde{X}$. This is in contrast with how fibre of $\mathcal{T} \tilde{X}$ is a sum of the fibre of $\mathcal{T} X$ and that of $\boldsymbol{\Delta}$.

In fact, a natural and simple lift of the normal bundle and its topological classes dictates

$$
\widehat{m(\mathcal{N})}=m(\tilde{\mathcal{N}}), \quad \widehat{e(\mathcal{N})}=e(\tilde{\mathcal{N}})
$$

In other words,

$$
\frac{1}{|W|} \int_{\tilde{X}} m(\mathcal{T} \tilde{X}) \wedge \frac{e(\tilde{\mathcal{N}})}{m(\tilde{\mathcal{N}})} \wedge \frac{e(\boldsymbol{\Delta})}{m(\boldsymbol{\Delta})}
$$

\footnotetext{
${ }^{1} \mathrm{The} \mathrm{ch}_{\xi}^{*}$ class of a bundle denotes the $\mathrm{ch}_{\xi}$ class of the dual bundle.
} 


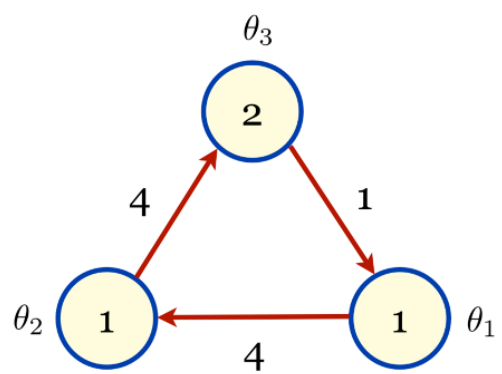

Figure 2. Non-Abelian triangular quiver with adjacency matrix (3.1).

computes the $M$-integral of the multiplicative class $m$ via its Abelianized D-term variety $\tilde{X}$ and the embedded $\tilde{M}$. Again, we will be working with toric varieties, so all quantities here can be straightforwardly read-off from $\tilde{\mathcal{Q}}$.

\section{Examples with a loop: triangular quivers}

Having seen the general prescription for computing the Higgs phase index, we shall illustrate it, in this section, with simplest examples with an oriented loop: the triangular quivers.

\subsection{A simplest non-Abelian triangular quiver}

Let us consider the quiver with the adjacency matrix

$$
A=\left(\begin{array}{ccc}
0 & 4 & -1 \\
-4 & 0 & 4 \\
1 & -4 & 0
\end{array}\right)
$$

and the dimension vector $\mathbf{d}=\left(r_{1}, r_{2}, r_{3}\right)=(1,1,2)$, as depicted in figure 2 . The computation of $\chi(M)$ is illustrated in the branch where $\theta_{1}<0$ and $\theta_{3}>0$ so that the single bi-fundamental field from node 3 to node 1 gets a zero VEV. Firstly, by imposing D-terms, one is led to the ambient variety $X=\mathbb{P}^{3} \times G r(2,4)$. The vacuum moduli space $M$ is then embedded in $X$ through the F-term, defined as a section of the rank-2 vector bundle associated with the vanishing $(\overline{\mathbf{2}}, \mathbf{1})$-bi-fundamental field under $\mathrm{U}(2)_{3} \times \mathrm{U}(1)_{1}$, where the subscripts for gauge groups label the nodes.

Now, we shall apply the general prescription of section 2 and move towards the territory of line bundles on toric geometry, as opposed to that of vector bundles on Grassmannian geometry. Upon Abelianizing the quiver, the rank-2 node gives rise to two Abelian nodes. Thus, the resulting quiver is described by the following adjacency matrix

$$
A=\left(\begin{array}{cccc}
0 & 4 & -1 & -1 \\
-4 & 0 & 4 & 4 \\
1 & -4 & 0 & 0 \\
1 & -4 & 0 & 0
\end{array}\right)
$$

with the dimension vector $\mathbf{d}=(1,1,1,1)$, as depicted in figure 3 . It is easy to see that the 


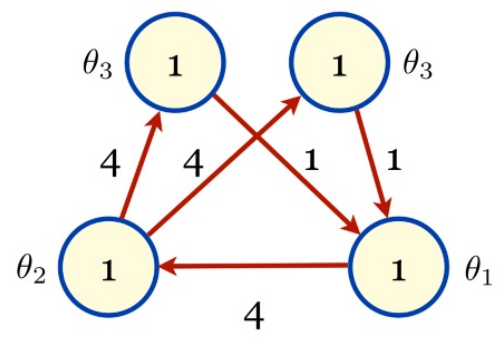

Figure 3. Abelian quiver with adjacency matrix (3.2), obtained through Abelianization of the quiver in figure 2.

corresponding D-term variety is $\tilde{X}=\mathbb{P}^{3} \times \mathbb{P}^{3} \times \mathbb{P}^{3}$. Note that the $G r(2,4)$ piece of the original D-term variety $X=\mathbb{P}^{3} \times G r(2,4)$ has led to the last two $\mathbb{P}^{3} \simeq G r(1,4)$ factors of $\tilde{X}$ upon Abelianization.

By taking the multiplicative class $m$ in eq. (2.27) to be the Chern class $c$, one is thus led to the following expression for $\chi(M)$ as an integral over $\tilde{X}=\mathbb{P}_{J}^{3} \times \mathbb{P}_{K_{1}}^{3} \times \mathbb{P}_{K_{2}}^{3}$,

$$
\begin{aligned}
\chi(M)= & \frac{1}{2} \int_{\tilde{X}} c(\mathcal{T} \tilde{X}) \wedge \frac{e(\tilde{\mathcal{N}})}{c(\tilde{\mathcal{N}})} \wedge \frac{e(\boldsymbol{\Delta})}{c(\boldsymbol{\Delta})} \\
= & \frac{1}{2} \int_{\mathbb{P}_{J}^{3} \times \mathbb{P}_{K_{1}}^{3} \times \mathbb{P}_{K_{2}}^{3} \underbrace{(1+J)^{4} \wedge\left(1+K_{1}\right)^{4} \wedge\left(1+K_{2}\right)^{4}}_{c(\mathcal{T} \tilde{X})}} \\
& \wedge \underbrace{\frac{\left(J+K_{1}\right) \wedge\left(J+K_{2}\right)}{\left(1+J+K_{1}\right) \wedge\left(1+J+K_{2}\right)}}_{e(\tilde{\mathcal{N}}) \wedge c(\tilde{\mathcal{N}})^{-1}} \wedge \underbrace{\frac{\left(K_{1}-K_{2}\right) \wedge\left(K_{2}-K_{1}\right)}{\left(1+K_{1}-K_{2}\right) \wedge\left(1+K_{2}-K_{1}\right)}}_{e(\boldsymbol{\Delta}) \wedge c(\boldsymbol{\Delta})^{-1}} \\
=\frac{1}{2} \oint & \mathrm{d} J \mathrm{~d} K_{1} \mathrm{~d} K_{2}\left(\frac{1+J}{J}\right)^{4}\left(\frac{1+K_{1}}{K_{1}}\right)^{4}\left(\frac{1+K_{2}}{K_{2}}\right)^{4} \\
& \cdot \frac{\left(J+K_{1}\right)\left(J+K_{2}\right)}{\left(1+J+K_{1}\right)\left(1+J+K_{2}\right)} \cdot \frac{\left(K_{1}-K_{2}\right)\left(K_{2}-K_{1}\right)}{\left(1+K_{1}-K_{2}\right)\left(1+K_{2}-K_{1}\right)},
\end{aligned}
$$

where $J, K_{1}$ and $K_{2}$ denote the Kähler classes of the three $\mathbb{P}^{3}$ factors, respectively, and in the last step, via the trivial intersection structure of $\mathbb{P}^{3}$, the integration of the cohomology class has switched to a contour integral around the origin. ${ }^{2}$ Note that the $(2 \pi i)^{-1}$ factor is implicit in each of the contour integral measures. It is straightforward to evaluate eq. (3.4) and we obtain $\chi(M)=12$.

As for the computation of the refined Euler character, we again apply eq. (2.27), now with $m=\mathrm{Td} \wedge \mathrm{ch}_{\xi}^{*}$,

$$
\chi_{\xi}(M)=\frac{1}{2} \int_{\tilde{X}} \operatorname{Td}(\mathcal{T} \tilde{X}) \wedge \operatorname{ch}_{\xi}\left(\mathcal{T}^{*} \tilde{X}\right) \wedge \frac{e(\tilde{\mathcal{N}})}{\operatorname{Td}(\tilde{\mathcal{N}}) \wedge \operatorname{ch}_{\xi}\left(\tilde{\mathcal{N}}^{*}\right)} \wedge \frac{e(\boldsymbol{\Delta})}{\operatorname{Td}(\boldsymbol{\Delta}) \wedge \operatorname{ch}_{\xi}\left(\boldsymbol{\Delta}^{*}\right)}
$$

\footnotetext{
${ }^{2}$ Note that we could have had $-J$ replacing $J$ in eq. (3.3) to conform with the convention used in subsection 3.2 for a general triangular quiver. Under such a choice, $J$ would not lie in the Kähler cone and eq. (3.4) should get an extra sign factor due to the negative intersection.
} 


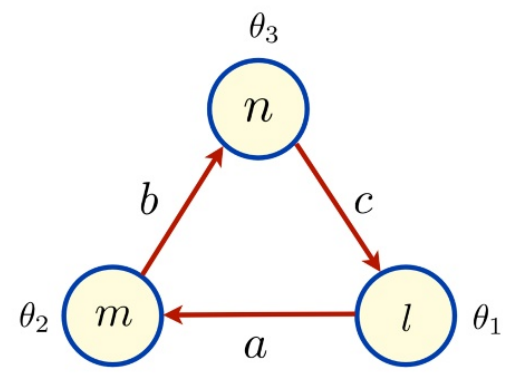

Figure 4. A general non-Abelian triangular quiver

where the four factors in the integrand are written in turn as

$$
\begin{aligned}
\operatorname{Td}(\mathcal{T} \tilde{X}) & =\left(\frac{J}{1-e^{-J}}\right)^{4}\left(\frac{K_{1}}{1-e^{-K_{1}}}\right)^{4}\left(\frac{K_{2}}{1-e^{-K_{2}}}\right)^{4} \\
\operatorname{ch}_{\xi}\left(\mathcal{T}^{*} \tilde{X}\right) & =\frac{1}{(1+\xi)^{3}}\left(1+\xi e^{-J}\right)^{4}\left(1+\xi e^{-K_{1}}\right)^{4}\left(1+\xi e^{-K_{2}}\right)^{4}, \\
\frac{e(\tilde{\mathcal{N}})}{\operatorname{Td}(\tilde{\mathcal{N}}) \wedge \operatorname{ch}_{\xi}\left(\tilde{\mathcal{N}}^{*}\right)} & =\frac{\left(1-e^{-J-K_{1}}\right)\left(1-e^{-J-K_{2}}\right)}{\left(1+\xi e^{-J-K_{1}}\right)\left(1+\xi e^{-J-K_{2}}\right)} \\
\frac{e(\boldsymbol{\Delta})}{\operatorname{Td}(\boldsymbol{\Delta}) \wedge \operatorname{ch}_{\xi}\left(\boldsymbol{\Delta}^{*}\right)} & =\frac{\left(1-e^{K_{1}-K_{2}}\right)\left(1-e^{K_{2}-K_{1}}\right)}{\left(1+\xi e^{K_{1}-K_{2}}\right)\left(1+\xi e^{K_{2}-K_{1}}\right)} .
\end{aligned}
$$

Similarly to the unrefined case, we are led to a straightforward contour integral and thereby obtain

$$
\chi_{\xi}(M)=1-2 \xi+3 \xi^{2}-3 \xi^{3}+2 \xi^{4}-\xi^{5},
$$

which gives the refined Higgs index

$$
\begin{aligned}
\Omega(y)[M] & =(-y)^{-d} \chi_{\xi=-y^{2}}(M) \\
& =-\frac{1}{y^{5}}-\frac{2}{y^{3}}-\frac{3}{y}-3 y-2 y^{3}-y^{5} .
\end{aligned}
$$

As desired, for $\xi=-1$, the refined Euler character (3.6) does reduce to the Euler number $\chi(M)=12$.

\subsection{General triangular quivers}

Let us now consider triangular quivers in full generality (see figure 4). In the previous example, the Abelian ambient variety $\tilde{X}$ was a product of projective spaces and hence, the integration (3.3) of a cohomology class, for instance, turned into the contour integral (3.4) in a trivial manner. In general, complications may arise due to the non-trivial intersection structure of $\tilde{X}$. We are still in the territory of toric geometry, however, and topological invariants can be obtained by some simple combinatorics. Let us work in the branch where the $c$ fields that transform as $(\overline{\mathbf{n}}, \mathrm{l})$ under $\mathrm{U}(\mathrm{n})_{3} \times \mathrm{U}(\mathrm{l})_{1}$ vanish simultaneously.

Figure 5 depicts the Abelianization of the quiver in figure 4. Note that the $c$ vanishing fields have been ignored for the simplicity of drawing. The D-term ambient space $\tilde{X}$ is 


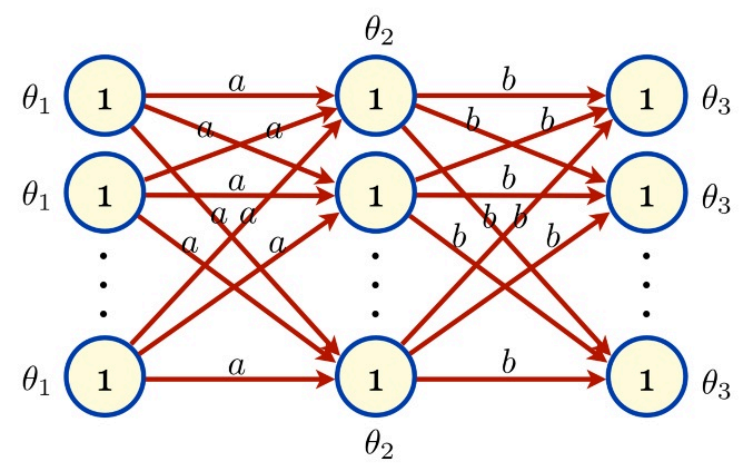

Figure 5. Abelianization of the quiver in figure 4; the $c$ vanishing fields are ignored here, given the branch in question.

a toric quiver variety and hence, can be completely described by its fan, which itself is determined by the charge matrix $Q$ together with $\theta$ values on the nodes (or the $\theta$-stability criterion). Amongst the $l+m+n$ Abelian groups, that is, $\mathrm{U}(1)_{\mathrm{A}, \mathrm{i}}$ for $i \in[1, l],[1, m],[1, n]$, respectively, for $A=1,2,3$, one can ignore an overall $\mathrm{U}(1)$ and we choose to take

$$
T / \mathrm{U}(1)=\prod_{\mathrm{i}=1}^{\mathrm{l}} \mathrm{U}(1)_{1, \mathrm{i}} \prod_{\mathrm{j}=1}^{\mathrm{m}} \mathrm{U}(1)_{2, \mathrm{j}} \prod_{\mathrm{k}=1}^{\mathrm{n}-1} \mathrm{U}(1)_{3, \mathrm{k}},
$$

with the last Abelian factor $\mathrm{U}(1)_{3, \mathrm{n}}$ quotiented from $T$.

Then, the $(l+m+n-1) \times(a m l+b m n)$ matrix $Q$, in an appropriate arrow ordering, can be written as follows:

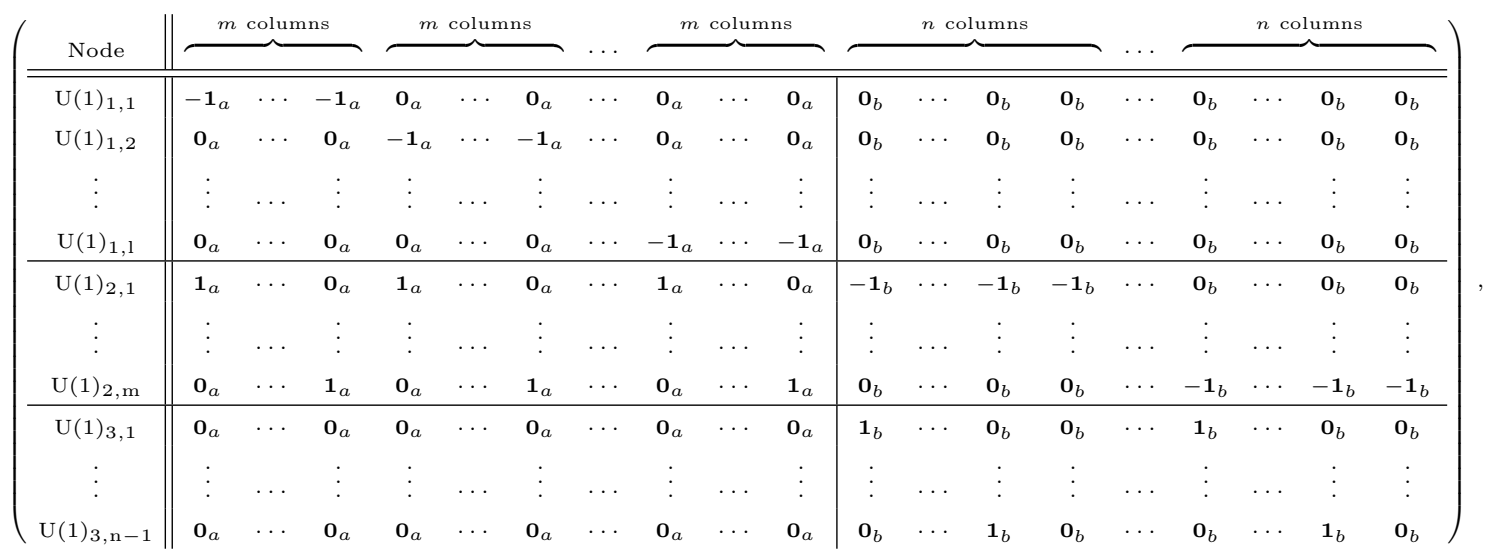

where $\mathbf{1}_{a}$ and $\mathbf{0}_{a}$ are row vectors of length $a$ with 1 and 0 in all directions, respectively, and similarly, $\mathbf{1}_{b}$ and $\mathbf{0}_{b}$ are row vectors of length $b$. Note that the redundant row associated with $\mathrm{U}(1)_{3, \mathrm{n}}$ has been removed and the matrix $Q$ consists only of $l+n+m-1$ rows. $^{3}$

To evaluate the Euler number, we apply eq. (2.27) with $m=c$,

$$
\chi(M)=\frac{1}{|W|} \int_{\tilde{X}} c(\mathcal{T} \tilde{X}) \wedge \frac{e(\tilde{\mathcal{N}})}{c(\tilde{\mathcal{N}})} \wedge \frac{e(\boldsymbol{\Delta})}{c(\boldsymbol{\Delta})}
$$

\footnotetext{
${ }^{3}$ Topological invariants do not depend on the choice of the row removal.
} 


$$
\begin{aligned}
= & \frac{1}{l ! m ! n !}\left[\int_{\tilde{X}}\right]_{L_{n}=0} \underbrace{\prod_{i=1}^{l} \prod_{j=1}^{m}\left(1-J_{i}+K_{j}\right)^{a} \wedge \prod_{j=1}^{m} \prod_{k=1}^{n}\left(1-K_{j}+L_{k}\right)^{b}}_{c(\mathcal{T} \tilde{X})} \\
& \wedge \underbrace{\prod_{i=1}^{l} \prod_{k=1}^{n}\left(\frac{-J_{i}+L_{k}}{1-J_{i}+L_{k}}\right)^{c}}_{e(\tilde{\mathcal{N}}) \wedge c(\tilde{\mathcal{N}})^{-1}} \\
& \wedge \underbrace{\prod_{i \neq i^{\prime}}^{l}\left(J_{i}-J_{i^{\prime}}\right) \wedge \prod_{j \neq j^{\prime}}^{m}\left(K_{j}-K_{j^{\prime}}\right) \wedge \prod_{k \neq k^{\prime}}^{n}\left(L_{k}-L_{k^{\prime}}\right)}_{e(\boldsymbol{\Delta}) \wedge c(\boldsymbol{\Delta})^{-1}}
\end{aligned}
$$

where $J_{i=1, \cdots, l}, K_{j=1, \cdots, m}$ and $L_{k=1, \cdots, n-1}$ are the Kähler forms arising from the three sets of U(1)'s, respectively. Note that for symmetry of integrand, a formal variable $L_{n}$ has been introduced, which should, in the end, be taken to vanish, as indicated by the integration symbol $\left[\int_{\tilde{X}}\right]_{L_{n}=0}$. Then, inserting the intersection numbers turns the integral (3.10) over the manifold $\tilde{X}$ to an equivalent contour integral around the origin, just as in eq. (3.4).

Similarly, the refined Euler character can be evaluated by applying eq. (2.27) with $m=\operatorname{Td} \wedge \operatorname{ch}_{\xi}^{*}$,

$$
\begin{aligned}
\chi_{\xi}(M)= & \frac{1}{|W|} \int_{\tilde{X}} \operatorname{Td}(\mathcal{T} \tilde{X}) \wedge \operatorname{ch}_{\xi}\left(\mathcal{T}^{*} \tilde{X}\right) \wedge \frac{e(\tilde{\mathcal{N}})}{\operatorname{Td}(\tilde{\mathcal{N}}) \wedge \operatorname{ch}_{\xi}\left(\tilde{\mathcal{N}}^{*}\right)} \wedge \frac{e(\boldsymbol{\Delta})}{\operatorname{Td}(\boldsymbol{\Delta}) \wedge \operatorname{ch}_{\xi}\left(\boldsymbol{\Delta}^{*}\right)} \\
= & \frac{1}{l ! m ! n !}\left[\int_{\tilde{X}}\right]_{L_{n}=0} \prod_{i=1}^{l} \prod_{j=1}^{m}\left(\frac{-J_{i}+K_{j}}{1-e^{J_{i}-K_{j}}}\right)^{a} \prod_{j=1}^{m} \prod_{k=1}^{n}\left(\frac{-K_{j}+L_{k}}{1-e^{K_{j}-L_{k}}}\right)^{b} \\
& \wedge \frac{1}{(1+\xi)^{l+m+n-1}} \prod_{i=1}^{l} \prod_{j=1}^{m}\left(1+\xi e^{-J_{i}+K_{j}}\right)^{a} \prod_{j=1}^{m} \prod_{k=1}^{n}\left(1+\xi e^{-K_{j}+L_{k}}\right)^{b} \\
& \wedge \prod_{i=1}^{l} \prod_{k=1}^{n}\left(\frac{1-e^{J_{i}-L_{k}}}{1+\xi e^{J_{i}-L_{k}}}\right)^{c} \\
& \wedge \prod_{i \neq i^{\prime}}^{l} \frac{1-e^{J_{i}-J_{i^{\prime}}}}{1+\xi e^{J_{i}-J_{i^{\prime}}}} \prod_{j \neq j^{\prime}}^{m} \frac{1-e^{K_{j}-K_{j^{\prime}}}}{1+\xi e^{K_{j}-K_{j^{\prime}}}} \prod_{k \neq k^{\prime}}^{n} \frac{1-e^{L_{k}-L_{k^{\prime}}}}{1+\xi e^{L_{k}-L_{k^{\prime}}}},
\end{aligned}
$$

where the integration symbol $\left[\int_{\tilde{X}}\right]_{L_{n}=0}$ means that the formal variable $L_{n}$ in the integrand is set to zero, as in the unrefined case (3.10).

For the rest of this section, we apply the index formulae (3.10) and (3.11) to two triangular, non-Abelian examples that illustrate mutation equivalence and non-trivial quiver invariant, respectively.

\subsubsection{Consistency check: quiver mutation}

Let us consider the non-Abelian quiver in figure 6 (left), in the branch where $\theta_{1}>0$ and $\theta_{2}<0$ so that the three arrows from node 1 to node 2 vanish. The topology of the 

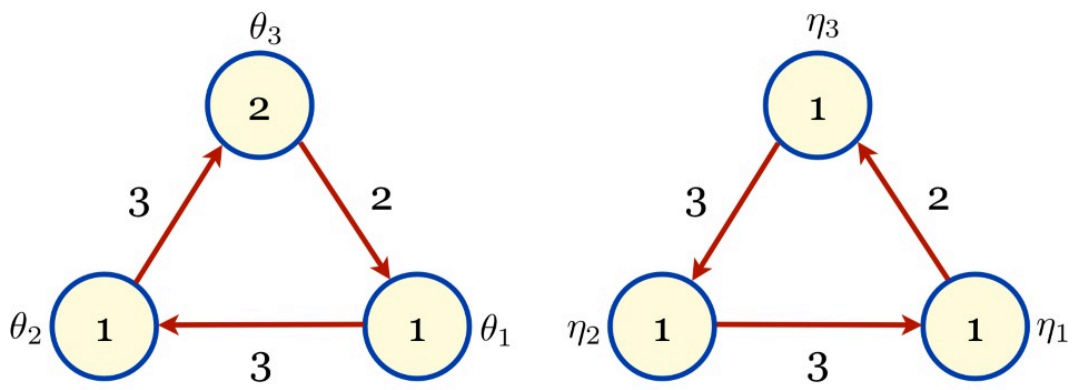

Figure 6. A non-Abelian triangular quiver (left) and an Abelian quiver (right); the former is obtained by mutating node 3 of the latter.

corresponding moduli space also depends on the sign of $\theta_{3}$ and we consider the case where $\theta_{3}>0$. The Euler number and the refined Euler character are in turn obtained by applying eqs. (3.10) and (3.11),

$$
\begin{aligned}
\chi(M) & =6, \\
\chi_{\xi}(M) & =1-4 \xi+\xi^{2} .
\end{aligned}
$$

On the other hand, the non-Abelian quiver in figure 6 on the left is mutation equivalent to the Abelian quiver on the right; the former in the given branch arises from mutating node 3 of the latter in the branch where $\eta_{1}>0$ and $\eta_{3}<0$ so that the two arrows from node 1 to node 3 vanish.

One can easily confirm that these are the correct branches by transforming the FI constants under the mutation,

$$
\begin{aligned}
& \theta_{1}=\eta_{1}, \\
& \theta_{2}=\eta_{2}+3 \eta_{3}, \\
& \theta_{3}=-\eta_{3} .
\end{aligned}
$$

The Abelian index computations turn out to give exactly the same results as in eqs. (3.12) and (3.13); this provides a consistency check for the non-Abelian indices as the two moduli spaces are mutation equivalent.

\subsubsection{Quiver invariants revisited}

Let us take the triangular quiver in figure 7 , which, due to the symmetry, has essentially two different branches: (a) $\theta_{1}<0, \theta_{3}>0$ where the three arrows from node 3 to node 1 vanish, and (b) $\theta_{1}>0, \theta_{2}<0$ where the five arrows from node 1 to node 2 vanish. The Euler number and the refined Euler character can be evaluated by applying eqs. (3.10) and (3.11),

$$
\begin{aligned}
& \chi\left(M_{a}\right)=6 \quad \text { and } \quad \chi\left(M_{b}\right)=9 \\
& \chi_{\xi}\left(M_{a}\right)=6, \quad \text { and } \quad \chi_{\xi}\left(M_{b}\right)=1-7 \xi+\xi^{2},
\end{aligned}
$$

in the branches (a) and (b), respectively. Consequently, we have the following refined Higgs phase indices

$$
\Omega_{\text {Higgs }}^{(a)}(y)=6 \text { and } \Omega_{\text {Higgs }}^{(b)}(y)=\frac{1}{y^{2}}+7+y^{2} .
$$




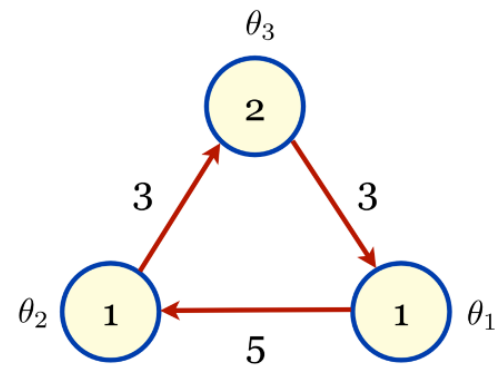

Figure 7. A simplest non-Abelian quiver with intrinsic Higgs states

The equivariant Coulomb phase indices, on the other hand, can be separately computed, along the lines of ref. [28, 29], as

$$
\Omega_{\text {Coulomb }}^{(a)}(y)=1+\Omega_{\text {Intrinsic }}^{(a)} \text { and } \Omega_{\text {Coulomb }}^{(b)}(y)=\frac{1}{y^{2}}+2+y^{2}+\Omega_{\text {Intrinsic }}^{(b)},
$$

where $\Omega_{\text {Intrinsic }}$ encodes the possibility of intrinsically Higgs states that cannot be directly counted via the Coulomb approach. By comparing eqs. (3.16) and (3.17), we see

$$
\Omega_{\text {Intrinsic }}^{(a)}=\Omega_{\text {Intrinsic }}^{(b)}=5=\Omega_{\text {Invariant }},
$$

and find that the notion of the intrinsic Higgs states as chamber-independent invariant of a quiver, first observed in Abelian quivers [16, 17, 30], manifests again in this non-Abelian example. ${ }^{4}$

\section{Abelianized Higgs index is a partition sum}

\subsection{Coulomb index as a partition sum}

With $\sum_{A} \gamma_{A}$ collection of BPS particles, with the intrinsic degeneracies $\Omega\left(\gamma_{A}\right)$ 's, the Coulomb index associated with the multi-particle BPS wavefunction can be computed as

$$
\begin{aligned}
\Omega^{-}\left(\sum \gamma_{A}\right)= & \Omega^{+}\left(\sum \gamma_{A}\right) \\
& +(-1)^{\sum_{A>B}\left\langle\gamma_{A}, \gamma_{B}\right\rangle+n-1} \frac{\prod_{A} \bar{\Omega}\left(\gamma_{A}\right)}{|\Gamma|} \int_{\mathcal{M}} \operatorname{ch}(\mathcal{F}) \wedge \mathcal{A}(\mathcal{M}) \\
& +(-1)^{\sum_{A^{\prime}>B^{\prime}}\left\langle\gamma_{A^{\prime}}^{\prime}, \gamma_{B^{\prime}}^{\prime}\right\rangle+n^{\prime}-1} \frac{\prod_{A^{\prime}} \bar{\Omega}\left(\gamma_{A^{\prime}}^{\prime}\right)}{\left|\Gamma^{\prime}\right|} \int_{\mathcal{M}^{\prime}} \operatorname{ch}\left(\mathcal{F}^{\prime}\right) \wedge \mathcal{A}\left(\mathcal{M}^{\prime}\right) \\
& +(-1)^{\sum_{A^{\prime \prime}>B^{\prime \prime}}\left\langle\gamma_{A^{\prime \prime}}^{\prime \prime}, \gamma_{B^{\prime \prime}}^{\prime \prime}\right\rangle+n^{\prime \prime}-1} \frac{\prod_{A^{\prime \prime}} \bar{\Omega}\left(\gamma_{A^{\prime \prime}}^{\prime \prime}\right)}{\left|\Gamma^{\prime \prime}\right|} \int_{\mathcal{M}^{\prime \prime}} \operatorname{ch}\left(\mathcal{F}^{\prime \prime}\right) \wedge \mathcal{A}\left(\mathcal{M}^{\prime \prime}\right) \\
& +\cdots
\end{aligned}
$$

where the charge $\sum_{A} \gamma_{A}$ is assumed to be primitive. $\Omega^{ \pm}$denote the indices on the two sides of marginal stability wall. When we cross the marginal stability wall, the Coulomb vacuum manifold $\mathcal{M}$ and its submanifolds $\mathcal{M}^{\prime}, \mathcal{M}^{\prime \prime}$, etc. become noncompact, and all the

\footnotetext{
${ }^{4} \Omega_{\text {Invariant }}=\Omega_{S}$ in the notation of refs. $[28,29]$, where these were left as an unknown input data.
} 
quantum ground states become non-normalizable. Only the first term $\Omega^{+}\left(\sum_{A} \gamma_{A}\right)$ may be present. This is how wall-crossing occurs from the Coulomb viewpoint.

The sum is over partitions of the total charge,

$$
\sum_{A=1}^{n} \gamma_{A}=\sum_{A^{\prime}=1}^{n^{\prime}} \gamma_{A^{\prime}}^{\prime}=\sum_{A^{\prime \prime}=1}^{n^{\prime \prime}} \gamma_{A^{\prime \prime}}^{\prime \prime}=\cdots
$$

such that $\gamma_{A^{\prime}}^{\prime}$ etc. are generally non-negative-integer linear combination of $\gamma_{A}$ 's. Note that $n>n^{\prime}$, meaning that in each of the partition, we have fewer number of particles than in the original problem. The barred $\Omega$ 's are the so-called rational invariant

$$
\bar{\Omega}(\gamma)=\sum_{p \mid \gamma} \Omega(\gamma / p) / p^{2}
$$

defined as a sum over all divisors of the charge in question. When $\sum_{A} \gamma_{A}$ is not primitive, it suffices to replace $\Omega^{ \pm}\left(\sum \gamma_{A}\right)$ by its rational counterpart. Finally $\Gamma^{\prime}$ is a set of permutation groups that mixes up identical charges among $\gamma_{A}^{\prime}$ 's. For further details of the formula, such as the nature of space $\mathcal{M}$ 's and the magnetic field strengths $\mathcal{F}$ 's, we direct readers to ref. [4].

This wall-crossing formula actually incorporates possibility of BPS states of the same charge arising from different constituent particles, i.e., from different quivers. For a single quiver, such a sum is due to the Weyl projection, or equivalently from the quantum statistics of indistinguishable particles. Starting with a particular quiver with a rank- $r_{v}$ node of a primitive charge $\gamma_{v}$, a partition, $r_{v}=\sum_{a_{v}=1}^{l_{v}} r_{v, a_{v}}$, contributes a term proportional to

$$
\frac{1}{\left|\Gamma\left(\left\{r_{v, a_{v}}\right\}\right)\right|} \prod_{a_{v}=1}^{l_{v}} \Omega\left(\gamma_{v}\right) / r_{v, a_{v}}^{2}
$$

where $\Gamma\left(\left\{r_{v, a_{v}}\right\}\right)$ is subgroup of the permutation group $S\left(r_{v}\right)$, or the Weyl group, that survives when some of $r_{v, a_{v}}$ 's equal. The effective moduli space $\mathcal{M}^{\prime}$ for such a partition is that of $l_{v}$ particles of charges $\gamma_{A^{\prime}}^{\prime}=r_{v, a_{v}} \gamma_{v}$ for $a_{v}=1, \cdots, l_{v}$, etc., which in turn is a submanifold of $\mathcal{M}$. The above general wall-crossing formula can be rebuilt from this by allowing different quivers contributing to the same charge states $[4] .^{5}$

Recall that, at least for cases without scaling regimes, the Higgs and the Coulomb answers have been shown to be equivalent $[1,3]$. This implies that Higgs index of some non-Abelian quivers can also be decomposed into a sum of indices of finitely many Abelian quivers, which are obtained by partitions of each non-Abelian nodes, say, of rank $r_{v}$, as $r_{v}=\sum_{a_{v}} r_{v, a_{v}}$. While quite natural and proven rigorously on the Coulomb side, the physical or mathematical origin of such a decomposition is quite opaque in the Higgs side. For the quivers with a loop and the scaling regime, furthermore, the equivalence between

\footnotetext{
${ }^{5}$ Ref. [12] also arrived at the same formula, again utilizing quantum statistics but in a rather different manner. In the approach of ref. [12], one factor $1 / p$ in the rational invariant arises from statistics while another $1 / p$ arises from the assumption that basic building blocks of the index are linear with respect to the pairwise Schwinger products. The latter assumption actually fails for quivers with scaling regime, just as the approach of ref. [4] cannot be trusted either in the presence of the scaling regime.
} 
Coulomb and Higgs sides no longer holds in general. Higgs side is more comprehensive and one finds states missed by the Coulomb "phase" computation. So one might wonder whether the partition sum representation is still possible for such quivers at all.

We wish to argue that our Abelianization routine for computing Higgs "phase" index naturally leads again to another sum over partitions of the total charge, which can be viewed as the Higgs counterpart of (4.1). In the next subsection, we shall study our Abelianization procedure and, for some simple cases without intrinsic Higgs states, compare the resulting partition sum with those found in Coulomb "phase" computation. The comparison will be made term by term and a complete agreement found for the examples.

\subsection{Is the Higgs index also a partition sum?}

Let us revisit the general Abelianization prescription for computing the refined Higgs index. In sections 2 and 3, we proposed and tested for some examples that it can be computed starting with the Cartan data of the quiver as

$$
\Omega(y)=\frac{1}{|W|} \int_{\tilde{X}} \omega_{y}(\mathcal{T} \tilde{X}) \wedge \frac{e(\tilde{\mathcal{N}})}{\omega_{y}(\tilde{\mathcal{N}})} \wedge \frac{e(\boldsymbol{\Delta})}{\omega_{y}(\boldsymbol{\Delta})},
$$

where $\tilde{M}$ and $\tilde{X}$ are the would-be Higgs moduli space and the ambient D-term variety, respectively, of the Abelianized quiver, and $\tilde{\mathcal{N}}$ is the normal bundle of $\tilde{M}$ embedded in $\tilde{X}$. The gauge group of the Abelianized quiver is the Cartan subgroup $T$ of the non-Abelian quiver but the chiral multiplet contents are kept intact. Note that for the Abelianized quiver, the refined index is given by integration of the first two factors in the integrand (4.2) without the last factor depending on $\boldsymbol{\Delta}$.

Recall that the vector bundle $\boldsymbol{\Delta}=\boldsymbol{\Delta}^{*}$ is the sum of line bundles, associated with "off-diagonal" part of the gauge group $G$,

$$
\bigoplus_{\alpha \in \Delta} \mathcal{L}_{\alpha}=\left(\bigoplus_{\alpha \in \Delta^{+}} \mathcal{L}_{\alpha}\right) \bigoplus\left(\bigoplus_{\alpha \in \Delta^{-}} \mathcal{L}_{\alpha}\right)
$$

with fiber $G / T$, which gives

$$
\frac{e(\boldsymbol{\Delta})}{\omega_{y}(\boldsymbol{\Delta})}=\prod_{\alpha \in \Delta} \frac{1-e^{-c_{1}\left(\mathcal{L}_{\alpha}\right)}}{y e^{-c_{1}\left(\mathcal{L}_{\alpha}\right)}-y^{-1}}=\prod_{\alpha \in \Delta^{+}}\left(1-\delta_{\alpha}\right),
$$

which defines $\delta_{\alpha}$ for each positive root $\alpha$ as

$$
\delta_{\alpha} \equiv \frac{\left(y-y^{-1}\right)^{2}}{y^{2}+y^{-2}-2 \cosh \left(c_{1}\left(\mathcal{L}_{\alpha}\right)\right)} .
$$

Note that the expression (4.3), when expanded, has $2^{\left|\Delta^{+}\right|}$terms of the form

$$
\delta(\mathcal{I}) \equiv(-1)^{|\mathcal{I}|} \prod_{\alpha \in \mathcal{I}} \delta_{\alpha},
$$

where $\mathcal{I} \subset \Delta^{+}$is a collection of positive roots. 


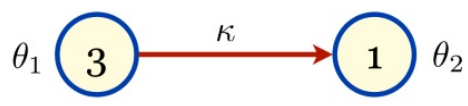

Figure 8. Grassmannian quiver with $\mathbf{d}=(3,1)$ and linking number $\kappa$

It is clear that the very first term in the expansion of (4.3), i.e., the term without any $\delta_{\alpha}$ factors, computes in (4.2) the Higgs index of the Abelianized quiver $\tilde{\mathcal{Q}}$, divided by $|W|$. Recall that we started with a quiver $\mathcal{Q}$ with the gauge group $\prod_{v=1}^{N} \mathrm{U}\left(\mathrm{r}_{\mathrm{v}}\right)$, where $v$ labels the $N$ nodes, and then obtained an Abelian quiver $\tilde{\mathcal{Q}}$ of rank $r+1=\sum_{v} r_{v}$ by replacing $\prod_{v} \mathrm{U}\left(\mathrm{r}_{\mathrm{v}}\right)$ in $\mathcal{Q}$ by $\prod_{v} \mathrm{U}(1)^{\mathrm{r}_{\mathrm{v}}}$ and maintaining the chiral field contents intact. $\tilde{M}$ is precisely the Higgs moduli space of $\tilde{\mathcal{Q}}$ in the chamber determined by $\theta$ 's inherited from $\mathcal{Q}$.

A given positive root $\alpha$ of $\prod_{v} \mathrm{U}\left(\mathrm{r}_{\mathrm{v}}\right)$ connects a distinct and unique pair of Cartan generators in $\prod_{v} \mathrm{U}(1)^{\mathrm{r}_{\mathrm{v}}}$, which tells us that for any given subset $\mathcal{I} \subset \Delta^{+}$we can associated an unordered partition $\mathcal{P}_{\mathcal{I}}$. One merely counts Cartan generators, connected pairwise by elements of $\mathcal{I}$, and call the resulting integers, $r_{v, a_{v}}$. Two extreme examples are the maximal partition,

$$
\mathcal{P}_{\phi}=\left(\{\underbrace{1,1, \cdots, 1}_{r_{1} \text { copies of } 1}\} ;\{\underbrace{1,1, \cdots, 1}_{r_{2} \text { copies of } 1}\} \cdots ;\{\underbrace{1,1, \cdots, 1}_{r_{N} \text { copies of } 1}\}\right)
$$

corresponding to the empty subset $\mathcal{I}=\phi$, and the minimal, or trivial, partition

$$
\mathcal{P}_{\Delta^{+}}=\left(\left\{r_{1}\right\},\left\{r_{2}\right\}, \cdots,\left\{r_{N}\right\}\right) .
$$

for $\mathcal{I}=\Delta^{+}$. Note that there are in general many different $\mathcal{I}$ 's that map to the same unordered partition $\mathcal{P}$.

For each (unordered) partition $\mathcal{P}$, we may associate an Abelian quiver $\mathcal{Q}_{\mathcal{P}}$. The Abelian quiver $\tilde{\mathcal{Q}}$ we encountered many time already is an example of this,

$$
\tilde{\mathcal{Q}}=\mathcal{Q}_{\mathcal{P}_{\phi}} .
$$

One way to picture $\mathcal{Q}_{\mathcal{P}}$ for a given $\mathcal{P}=\left(\left\{r_{v, a_{v}}\right\}\right)$ is to start with $\tilde{\mathcal{Q}}=\mathcal{Q}_{\mathcal{P}_{\phi}}$, gather $\mathrm{U}(1)$ nodes of $\tilde{\mathcal{Q}}$ according to the numbers $\left\{r_{v, a_{v}}\right\}$, and fuse each such collection to a single Abelian node, of which FI constant is given by summing those of the fused nodes. In terms of the Coulomb side picture, such a node corresponds to a single center of (non-primitive) charge $r_{v, a_{v}} \gamma_{v}$. Naturally $\mathcal{Q}_{\mathcal{P}}$ has the gauge group $\prod_{v} \prod_{a_{v}} \mathrm{U}(1)$, typically of smaller rank than $r+1=\sum_{v} r_{v}=\sum_{v} \sum_{a_{v}} r_{v, a_{v}}$. We keep the bi-fundamental chiral field contents intact, which is accomplished as the intersection numbers are multiplied by a pair of $r_{v, a_{v}}$ 's in an obvious manner:

$$
r_{v, a_{v}} \times\left\langle\gamma_{v}, \gamma_{w}\right\rangle \times r_{w, b_{w}} .
$$

As an illustration of the mapping $\mathcal{P} \mapsto \mathcal{Q}_{\mathcal{P}}$, let us consider the Grassmannian quiver in figure 8, with gauge group $G=\mathrm{U}(3) \times \mathrm{U}(1)$, that has the dimension vector $\mathbf{d}=(3,1)$. The dimension vector admits the following three partitions,

$$
\begin{aligned}
& \mathcal{P}_{1}=(\{1,1,1\} ;\{1\}), \\
& \mathcal{P}_{2}=(\{1,2\} ;\{1\}), \\
& \mathcal{P}_{3}=(\{3\} ;\{1\}),
\end{aligned}
$$



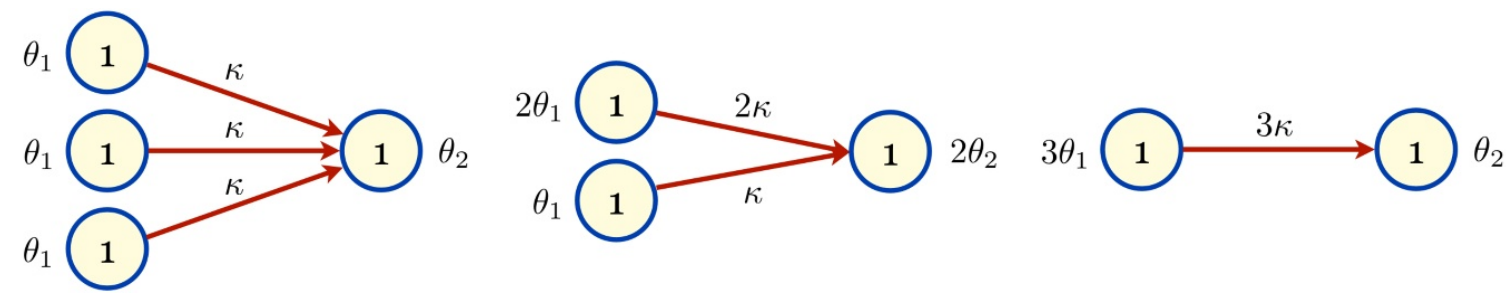

Figure 9. The three Abelian quivers arising from the non-Abelian quiver in figure 8; they correspond, respectively(from left to right), to the three partitions $\mathcal{P}_{i}$ for $i=1,2,3$ given in eq. (4.8).

which, according to the rule explained in the previous paragraph, correspond, respectively, to the three quivers depicted in figure 9 .

To state the conjecture, we now come back to the contribution from $\boldsymbol{\Delta}$ to the refined Higgs index. Reorganizing the terms as

$$
\frac{e(\boldsymbol{\Delta})}{\omega_{y}(\boldsymbol{\Delta})}=\prod_{\alpha \in \Delta^{+}}\left(1-\delta_{\alpha}\right)=\sum_{\mathcal{P}} \sum_{\mathcal{P}_{\mathcal{I}}=\mathcal{P}, \mathcal{I} \subset \Delta^{+}} \delta(\mathcal{I}),
$$

we have a sum over partitions for the refined index as

$$
\Omega(y)[\mathcal{Q}]=\sum_{\mathcal{P}}\left(\frac{1}{|W|} \int_{\tilde{X}}\left[\omega_{y}(\mathcal{T} \tilde{X}) \wedge \frac{e(\tilde{\mathcal{N}})}{\omega_{y}(\tilde{\mathcal{N}})} \wedge \sum_{\mathcal{P}_{\mathcal{I}}=\mathcal{P}, \mathcal{I} \subset \Delta^{+}} \delta(\mathcal{I})\right]\right) .
$$

For a general quiver, we claim that each and every term in the sum over $\mathcal{P}$ represents contribution from the Abelian quiver $\mathcal{Q}_{\mathcal{P}}$ defined above.

As we noted earlier, a partition sum of similar kind has been rigorously demonstrated on the Coulomb side computation, which is reliable for quivers without loops. As the Higgs index and the Coulomb index equal in these cases, a partition sum does already exist in the Higgs side as well. What we claim is that, for such general quivers, our partition sum coincides exactly and term-by-term with this physically motivated partition sum. Borrowing from these works, then, our conjecture can be stated as

$$
\frac{1}{|W|} \int_{\tilde{X}}\left[\omega_{y}(\mathcal{T} \tilde{X}) \wedge \frac{e(\tilde{\mathcal{N}})}{\omega_{y}(\tilde{\mathcal{N}})} \wedge \sum_{\mathcal{P}_{\mathcal{I}}=\mathcal{P}, \mathcal{I} \subset \Delta^{+}} \delta(\mathcal{I})\right]=c(\mathcal{P} ; y) \times \Omega(y)\left[Q_{\mathcal{P}}\right]
$$

for each unordered partition $\mathcal{P}$, where

$$
c(\mathcal{P} ; y) \equiv \frac{1}{|\Gamma(\mathcal{P})|} \prod_{v=1}^{N} \prod_{a_{v}=1}^{l_{v}} \frac{1}{r_{v, a_{v}}} \frac{y-y^{-1}}{y^{r_{v, a_{v}}}-y^{-r_{v, a_{v}}}},
$$

is a well-established universal factor that appears in the Coulomb phase wall-crossing formula. For the nonequivariant limit, terms in the product here reduce to $\pm 1 / r_{v, a_{v}}^{2}$ we already encountered at the top of this section. Although $c(\mathcal{P} ; y)$ was found in the study of quivers without loops, its origin lies entirely in the quantum statistics or equivalently the Weyl groups and the same formula should be applicable to general quivers. 
Again, to illustrate our proposal for the new partition-sum structure, let us revisit the Grassmannian example in figure 8. Firstly, the gauge group $G=\mathrm{U}(3) \times \mathrm{U}(1)$ has three positive roots, which we may denote as $\Delta^{+}=\left\{\alpha_{12}, \alpha_{13}, \alpha_{23}\right\}$. Then, for instance, the subset $\mathcal{I}=\left\{\alpha_{12}\right\} \subset \Delta^{+}$maps to the partition $\mathcal{P}_{\mathcal{I}}=(\{1,2\} ;\{1\})$ and hence, to the second quiver in figure 9 , as the two out of the three nodes arising from the rank-3 node of the original non-Abelian quiver, are fused together via the single element $\alpha_{12}$ in $\mathcal{I}$. It is easy to see that the eight subsets of $\Delta^{+}$can be grouped into the following three,

$$
\begin{aligned}
& \mathcal{I}_{1}=\phi \\
& \mathcal{I}_{2}=\left\{\alpha_{12}\right\},\left\{\alpha_{13}\right\},\left\{\alpha_{23}\right\} \\
& \mathcal{I}_{3}=\left\{\alpha_{12}, \alpha_{13}\right\},\left\{\alpha_{12}, \alpha_{23}\right\},\left\{\alpha_{13}, \alpha_{23}\right\},\left\{\alpha_{12}, \alpha_{13}, \alpha_{23}\right\}
\end{aligned}
$$

so that $\mathcal{I}_{i}$ map to $\mathcal{P}_{i}$ for $i=1,2,3$ and, in turn, to the three quivers in figure 9 , respectively (from left to right). Our conjecture (4.11) asserts, for instance, that the left hand side, which is a sum over the three subsets $\mathcal{I}_{2}$ in eq. (4.13), should equal the Higgs phase index on the right hand side for the second quiver in figure 9, multiplied by the appropriate prefactor (4.12), which in this case is

$$
c\left(\mathcal{P}_{\mathcal{I}_{2}} ; y\right)=\frac{1}{2} \frac{1}{y+y^{-1}} .
$$

To summarize, we rewrote the Abelianization formula for refined Higgs index in terms of a partition sum. Each summand, labeled by $\mathcal{P}$, is then conjectured to compute refined Higgs index of a specific Abelian quiver $Q_{\mathcal{P}}$, up to universal factor $c(\mathcal{P} ; y)$. As noted many times, quivers without loops are known to admit an expansion via partition of the total charge, and this was motivated, tested, and proven in the Coulomb description and thus is trustworthy for such quivers. What our computation and the conjecture suggests, as we will see below for the simplest classes of non-Abelian quiver, is that (4.10) coincides with this existing partition sum via eq. (4.11), even though our partition-sum expansion comes from an entirely mathematical manifestation in the Higgs description.

The real substance of this conjecture lies in that this phenomenon holds for general quivers with loops as well. As the Abelianization procedure works in the presence of Fterms, the partition sum (4.10) clearly holds as a mathematical statement, yet, whether eq. (4.11) holds is hardly clear, a priori. For general quivers with loop, another conjectural form of such a partition sum has been proposed by Manschot, Pioline, and Sen [28, 29]. However, this leaves behind the counting of the so-called intrinsic Higgs states as undetermined input data. Our formulae compute the Higgs index, including contributions from the quiver invariants directly, and thus offer a self-complete routine for counting BPS states.

Two-node quivers. Let us prove the conjecture (4.11) for the Grassmannian quivers, consisting of an Abelian and a rank- $r$ nodes connected by $\kappa$ bi-fundamental fields. See figure 8 for the case of $r=3$. The Higgs moduli space is $\operatorname{Gr}(r, \kappa)$. The formula (4.2) is expanded as

$$
\Omega(y)[G r(r, \kappa)]=\frac{1}{|W|} \int_{\tilde{X}}\left[w_{y}(\mathcal{T} \tilde{X}) \wedge\left(\sum_{\mathcal{I} \subset \Delta^{+}} \delta(\mathcal{I})\right)\right]
$$


where $\delta(\mathcal{I})$ 's are as defined in eqs. (4.5) and (4.4):

$$
\delta(\mathcal{I})=(-1)^{|\mathcal{I}|} \prod_{\alpha \in \mathcal{I}} \delta_{\alpha} \quad \text { with } \quad \delta_{\alpha}=\frac{\left(y-y^{-1}\right)^{2}}{y^{2}+y^{-2}-2 \cosh \left(c_{1}\left(\mathcal{L}_{\alpha}\right)\right)} .
$$

Note that the normal bundle pieces are missing as the quivers are tree-like. More explicitly, by introducing the $r$ Kähler forms $J_{i=1, \cdots, r}$ of $\tilde{X}=\left(\mathbb{P}^{\kappa-1}\right)^{r}$, the expansion (4.15) can be rewritten as

$$
\begin{aligned}
& \Omega(y)[G r(r, \kappa)] \\
= & \frac{1}{|W|} \frac{1}{\left(y-y^{-1}\right)^{r}} \int_{\tilde{X}}\left[\prod_{i=1}^{r}\left(J_{i} \wedge \frac{y e^{-J_{i}}-y^{-1}}{1-e^{-J_{i}}}\right)^{\kappa}\right] \wedge\left[\sum_{\mathcal{I} \subset \Delta^{+}} \delta(\mathcal{I})\right] \\
= & \frac{1}{|W|} \frac{1}{\left(y-y^{-1}\right)^{r}} \oint\left[\prod_{i=1}^{r} \mathrm{~d} J_{i}\right]\left[\prod_{i=1}^{r}\left(\frac{y e^{-J_{i}}-y^{-1}}{1-e^{-J_{i}}}\right)^{\kappa}\right]\left[\sum_{\mathcal{I} \subset \Delta^{+}} \delta(\mathcal{I})\right],
\end{aligned}
$$

where the contour integrals are around the circles centered at origin and the $(2 \pi i)^{-1}$ factor is implicit in each measure $\mathrm{d} J_{i}$. Our conjecture states that terms in the final sum are associated, via (4.11), with Abelian quivers $\mathcal{Q}_{\mathcal{P}}$ labeled by partition $\mathcal{P}$ of $(r, 1)$; for $r=3$, three such Abelian quivers are found in figure 9.

A simplest way to verify the partition-sum structure (4.11) is to consider the decomposition inductively on the rank $r$ of the non-Abelian node:

- For $r=1$ case, $\Delta^{+}$is empty and thus the factor $\sum_{\mathcal{I C} \subset \Delta^{+}} \delta(\mathcal{I})$ in the integrand of eq. (4.15) only has a single term, the unity, leading to the refined Euler character of $X(=\tilde{X})$ itself.

- For $r=2$ case, $\Delta^{+}=\left\{\alpha_{12}\right\}$ is a singleton with the unique positive root $\alpha_{12}$ of $\mathrm{U}(2)$ and the factor $\sum_{\mathcal{I} \subset \Delta^{+}} \delta(\mathcal{I})$ in eq. (4.15) has two terms, $\delta(\phi)=1$ and $\delta\left(\Delta^{+}\right)=$ $-\delta_{\alpha_{12}}$. The former corresponds exactly to eq. (4.11) for the maximal partition $\mathcal{P}=$ $(\{1,1\} ;\{1\})$ with $c(\mathcal{P} ; y)=\frac{1}{|W|}$. One can go brute force and also verify explicitly that the latter corresponds to eq. (4.11) for the minimal partition $\mathcal{P}=(\{2\} ;\{1\})$. There is a simpler way around to check this, however. Note first that the both sides of relation (4.11), when summed over all partitions $\mathcal{P}$, lead to one and the same invariant, the refined index of $G r(r, \kappa)$. As we have already checked the validity of relation (4.11) for the maximal partition out of the two available partitions, it should also hold for the remaining, minimal partition.

- Let us suppose that the relation (4.11) holds for all Grassmannian quivers with rank of the non-Abelian node less than $r$ and consider the $G r(r, \kappa)$ quiver. Since the first factor

$$
\prod_{i=1}^{r}\left(\frac{y e^{-J_{i}}-y^{-1}}{1-e^{-J_{i}}}\right)^{\kappa}
$$

of the integrand in eq. (4.16) factorizes to $r$ pieces, each depending on a single $J_{i}$ variable, the inductive assumption can be used to show that the relation (4.11) holds 


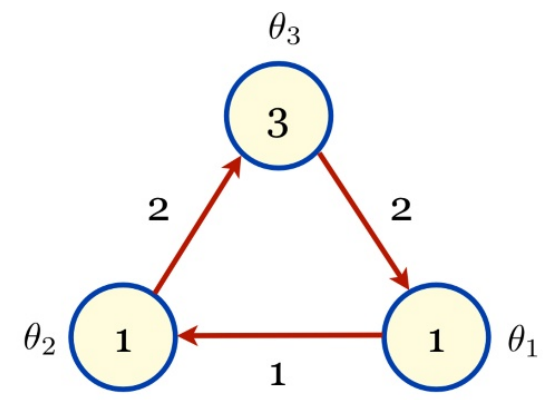

Figure 10. A non-Abelian quiver with a rank-3 node and two Abelian nodes
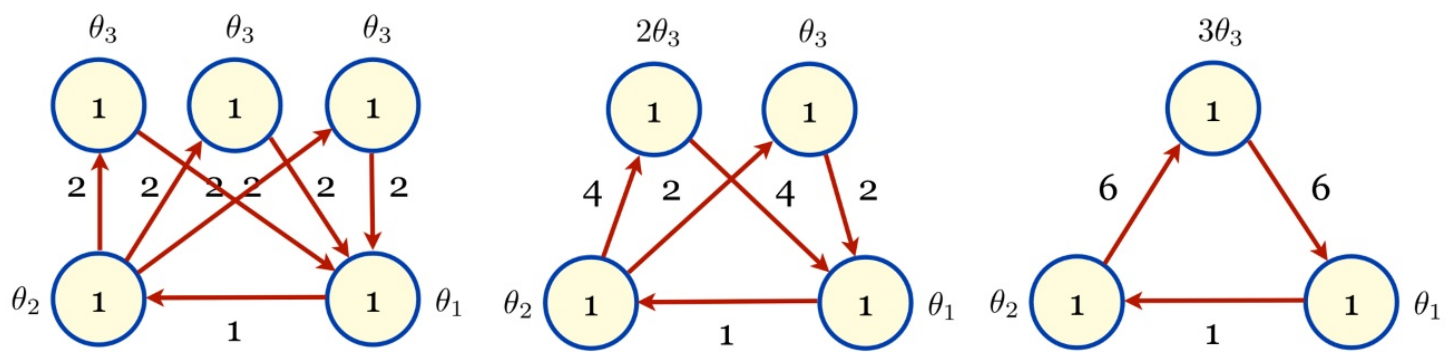

Figure 11. The three Abelian quivers arising from the non-Abelian quiver in figure 10; they correspond, respectively(from left to right), to the three partitions $\mathcal{P}_{i}$ for $i=1,2,3$ given in eq. (4.17).

for all partitions except possibly for the minimal one, $\mathcal{P}=(\{r\} ;\{1\})$. Then we may apply the same argument illustrated above for $r=2$ case: since the both sides of (4.11) sum up to a common invariant and the relation (4.11) is valid individually for all but the minimal partition, it should also hold for the minimal partition.

A simple non-Abelian cyclic quiver. as a next example, we take a non-Abelian cyclic and triangular quiver in figure 10. For the simplest such case, with rank 2 instead of rank 3 node at the top, the conjectured identity (4.11) holds somewhat trivially, once that it holds for $\mathcal{P}_{\phi}$ term, because there are only two possible partitions. Rank 3 case therefore offers the first nontrivial test. Also this case is a significant departure from the Grassmannian example above, because the quiver in question comes with a loop, and therefore the quiver dynamics has a superpotential.

We consider the branch where $\theta_{1}>0$ and $\theta_{2}<0$ so that the single bi-fundamental field from node 1 to node 2 vanishes. Following the Abelianization prescription, we first obtain the Abelianized quiver; see the first diagram in figure 11. Although the corresponding Dterm variety $\tilde{X}$ is still toric, its intersection structure is not as trivial as that of projective spaces (or products thereof). Now, the three quivers in figure 11 correspond, respectively, to the three partitions

$$
\begin{aligned}
& \mathcal{P}_{1}=(\{1\} ;\{1\} ;\{1,1,1\}), \\
& \mathcal{P}_{2}=(\{1\} ;\{1\} ;\{1,2\}), \\
& \mathcal{P}_{3}=(\{1\} ;\{1\} ;\{3\}),
\end{aligned}
$$


which, in turn, can be obtained from the subsets

$$
\begin{aligned}
& \mathcal{I}_{1}=\phi \\
& \mathcal{I}_{2}=\left\{\alpha_{12}\right\},\left\{\alpha_{13}\right\},\left\{\alpha_{23}\right\}, \\
& \mathcal{I}_{3}=\left\{\alpha_{12}, \alpha_{13}\right\},\left\{\alpha_{12}, \alpha_{23}\right\},\left\{\alpha_{13}, \alpha_{23}\right\},\left\{\alpha_{12}, \alpha_{13}, \alpha_{23}\right\},
\end{aligned}
$$

of the positive-root set $\Delta^{+}=\left\{\alpha_{12}, \alpha_{13}, \alpha_{23}\right\}$. Note that several subsets $\mathcal{I} \subset \Delta^{+}$can correspond to a given partition $\mathcal{P}$ in general.

In order to verify the desired partition-sum structure, the two sides of eq. (4.11) need to be computed for every partition of $\mathbf{d}=(1,1,3)$. We have indeed computed the lefthand-sides, using our Abelianization procedure, and find that these match precisely the right-hand-sides, respectively. For the record, we list them here;

$$
\begin{aligned}
& c\left(\mathcal{P}_{1} ; y\right) \times \Omega(y)\left[Q_{\mathcal{P}_{1}}\right]=-\frac{1}{6} y^{-7}-\frac{2}{3} y^{-5}-\frac{7}{6} y^{-3}-\frac{4}{3} y^{-1}-\frac{4}{3} y-\frac{7}{6} y^{3}-\frac{2}{3} y^{5}-\frac{1}{6} y^{7}, \\
& c\left(\mathcal{P}_{2} ; y\right) \times \Omega(y)\left[Q_{\mathcal{P}_{2}}\right]=\frac{1}{2} y^{-7}+y^{-5}+\frac{3}{2} y^{-3}+2 y^{-1}+2 y+\frac{3}{2} y^{3}+y^{5}+\frac{1}{2} y^{7}, \\
& c\left(\mathcal{P}_{3} ; y\right) \times \Omega(y)\left[Q_{\mathcal{P}_{2}}\right]=-\frac{1}{3} y^{-7}-\frac{1}{3} y^{-5}-\frac{1}{3} y^{-3}-\frac{2}{3} y^{-1}-\frac{2}{3} y-\frac{1}{3} y^{3}-\frac{1}{3} y^{5}-\frac{1}{3} y^{7} .
\end{aligned}
$$

The conjectured relation (4.11) is thus confirmed for this cyclic example.

\section{Acknowledgments}

We are grateful to Jaemo Park for collaboration at an early stage of this work and to Bumsig Kim for kind explanation of his works. We also thank Tarig Abdelgadir, Jan Manschot, Ashoke Sen, and Dan Xie for useful conversations. P.Y. is grateful to Institute for Advanced Study, Columbia University, and Perimeter Institute for hospitality while this work was in progress. This work is supported in part (P.Y.) by the National Research Foundation of Korea (NRF) via the Center for Quantum Spacetime (grant number 20050049409), and also (Z.L.W.) by National Natural Science Foundation of China with grant No.11305125.

\section{A Intersection structures of toric varieties}

To evaluate integrals such as (2.1) and (2.24), we still need to determine the various intersection numbers, which can only be understood through the complete fan structure for $\tilde{X}$. First of all, associated with the Abelianized quiver is a corresponding "charge matrix", denoted by $Q=\left[Q_{v e}\right]$, each row of which lists the charges of the bi-fundamental fields under each $\mathrm{U}(1)$ gauge group. Note that the row and the column indices for the matrix range over the regions $1 \leq v \leq \operatorname{rk}(T / \mathrm{U}(1)) \equiv \mathrm{r}$ and $1 \leq e \leq\left|\Sigma^{(1)}\right| \equiv k$, respectively. The charge matrix itself has a certain amount of information on the toric variety $\tilde{X}$. For instance, any multiplicative class of the tangent bundle $\mathcal{T} \tilde{X}$, say, the Chern class of $\mathcal{T} \tilde{X}$, can be expressed explicitly in terms of $Q$,

$$
c(\mathcal{T} \tilde{X})=\prod_{e=1}^{k}\left[1+\sum_{v=1}^{r} Q_{v e} J_{v}\right],
$$


where $J_{v=1, \cdots, r}$ form a basis of the $H^{2}(\tilde{X})$, which turns out to be of rank $r$. However, the charge matrix does not uniquely determine the fan; in toric terms, its rows correspond to the linear relations of the rays in the fan, but the incidence information for higher-dimensional cones is missing.

Let us now summarize how the complete fan structure for the toric variety $\tilde{X}$ is determined from the given quiver data, and also present the recipe for the intersection numbers. The technical details will not be needed in reading this paper and the way we state the procedures here is by no means pedagogical. Interested readers are kindly referred to the excellent maths texts [31-34] for a more complete review (see also, for instance, section 5 of the lecture note [35] for some concrete examples).

It turns out that the charge matrix, when equipped with $\theta$ values assigned to the quiver nodes (that is, a choice of $\theta$-stability criterion), does determine the fan completely; the notion of stability of reduced quivers can be defined accordingly, from which the fan structure is determined [26]. Practically, however, the procedure illustrated in ref. [27] can be more accessible, which goes as follows: an index set $\mathcal{A}$ is defined as

$$
\mathcal{A}=\left\{I \subset \Sigma^{(1)} \mid \exists a_{e}>0 \text { such that } \theta_{v}=\sum_{e \in I} Q_{v e} a_{e}, \text { for } 1 \leq v \leq r\right\},
$$

and by collecting the maximal elements of the complement $\mathcal{A}^{c}$ inside the power set of $\Sigma^{(1)}$, one obtains the Stanley-Reisner ideal $\mathcal{I}_{\mathrm{SR}}$, from which the corresponding fan $\Sigma$ is constructed as ${ }^{6}$

$$
\Sigma=\left\{I \subset \Sigma^{(1)} \mid I \not \supset S, \text { for all } S \in \mathcal{I}_{\mathrm{SR}}\right\} .
$$

Now given the charge matrix $Q$ and the fan $\Sigma$ for the toric variety $\tilde{X}$, the intersection numbers defined as

$$
\kappa_{v_{1} v_{2} \cdots v_{d}} \equiv \int J_{v_{1}} \wedge \cdots \wedge J_{v_{d}}, \quad \text { with } \quad 1 \leq v_{s=1, \ldots, d} \leq r,
$$

are determined by simultaneously solving the linear equations of the following form,

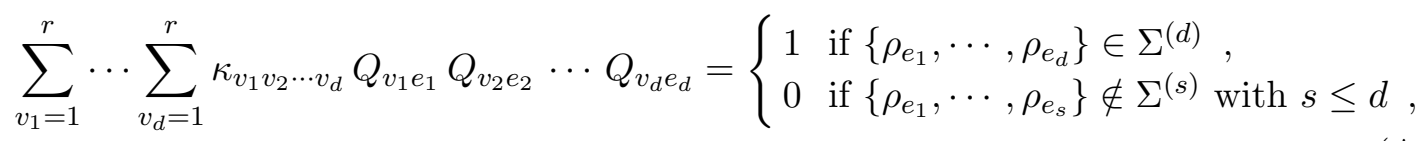

where $\rho_{e}$ denote the ray corresponding to the $e$-th column of $Q$, and $\Sigma^{(s)} \subset \Sigma$, the collection of $s$-dimensional cones for $1 \leq s \leq d$.

\section{B The ambient D-term variety and maximal reduced quivers}

For quivers with (oriented) loops, i.e. with superpotentials, the Higgs moduli space is determined by combining D-term and F-term constraints. Since the number of F-terms equals to the number of chiral fields participating in the superpotential, one might think that the vacua are at most point-like as far as these chiral fields are concerned, or more likely null if D-terms impose further nontrivial condition. However, this is not true; there

\footnotetext{
${ }^{6}$ By abuse of notation we denote the cone $\sigma=\operatorname{Span}(I) \subset \mathbb{R}^{d}$ simply by the set, $I$, of its generators.
} 
typically exists Higgs branches with nonnegative dimensions, which arises by setting entire bi-fundamentals between certain pairs of nodes identically zero. This kills many F-terms so effectively that the naive dimension counting above becomes irrelevant.

When this happens (and this happens generically for quivers with loops), the Higgs moduli space $M$ is embedded, via the surviving F-term conditions, in certain D-term quiver variety $X$. The quiver whose Higgs moduli space is $X$ is related to the original quiver by removal of certain edges so that the final quiver has no loop whatsoever. $X$ defines the ambient variety we have used throughout this paper. For the purpose of this appendix, we call these quivers without loops, obtained from the original quiver by removal of edges, "reduced quivers." We will presently claim that, with generic superpotentials and FI constants, the reduced quiver has to be always maximal in that restoration of any one edge would reintroduce a loop. This shows that $X$ is always a quiver variety obtained by symplectic reduction of higher dimensional flat complex vector space, $\mathbb{C}^{K}$, where $K$ is the total number of chiral superfields in the maximal reduced quiver.

In this appendix, we shall use $\vec{\Phi}^{s \bar{t}}=\left\{\Phi_{i_{s}}^{a_{s} \bar{b}_{t}}\right\}$ denoting the bi-fundamental fields associated with the edge connecting the node $s$ and $t$, where $a_{s}$ and $\bar{b}_{t}$ are the gauge indices and $i_{s \bar{t}}$ is the flavor index.

Firstly, let us recall the argument in ref. [17] for the Abelian cyclic quivers. For an $N$-node Abelian cyclic quiver, we only have the $N$ complex vectors $\vec{\Phi}^{s} \equiv \vec{\Phi}^{s, \overline{s+1}}$ for $s=1, \cdots, N$, with $\vec{\Phi}^{N}=\vec{\Phi}^{N, \overline{N+1}} \equiv \vec{\Phi}^{N, \overline{1}}$ understood. We can show that there is no solution to F-term conditions with all the complex vectors nontrivial. If there were such a solution, it has to be a discrete solution since the number of F-term equations equals the total number of complex variables $\vec{\Phi}^{s}$ s if none of the $\vec{\Phi}^{s}$ is vanishing. (We always assume that the coefficients in the superpotential $W=W\left(\left\{\Phi_{i_{s}}^{s}\right\}\right)$ are generic, so the algebraic equations $\partial W=0$ are also generic.) However, the above F-term conditions have $N$ scaling symmetries under $\vec{\Phi}^{s} \rightarrow \lambda_{s} \vec{\Phi}^{s}$ for any complex numbers $\lambda_{s}$, and one can actually generate $N$ complex dimensional family of solutions. This contradicts the expected discreteness of the solution, so we cannot generically expect to find solutions of this type.

Combining with the D-term conditions, one can show [17] the ambient D-term variety in each branch is related to a certain reduced quiver with one edge eliminated. ${ }^{7}$ In general, the eliminated edge is uniquely decided if the FI parameters are given. The removal of more than one edges only happens on the marginal stability wall.

For the Abelian multi-loop case, one can show similarly that the ambient D-term variety is determined entirely by a reduced quiver without loops. As before, if there is a solution to F-term conditions with all the complex vectors nonvanishing, it has to be a discrete solution for a generic choice of the superpotential since the number of F-term equations equals the total number of complex variables $\vec{\Phi}^{s \bar{t}}$ 's which appear in the superpotential. Since the full set of F-term equations are not homogenous now, we can not use the previous argument to generated a family of solutions. However, for the quivers we are interested in, there is a $\mathrm{U}(1)_{\mathrm{R}}$ symmetry under which the superpotential has charge 2 . This

\footnotetext{
${ }^{7}$ By abuse of notation, in this appendix, an edge means the whole bunch of arrows between a pair of nodes.
} 


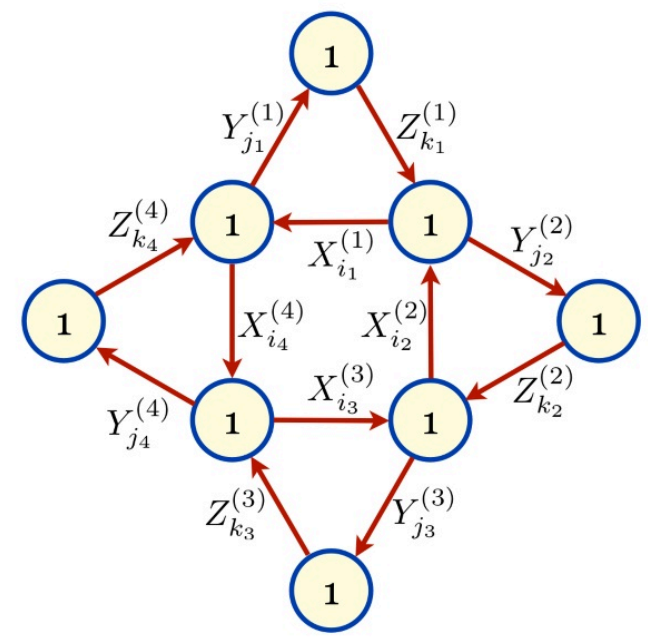

Figure 12. Abelian quiver with total of 6 loops; the linking numbers and the FI constants are implicit, while the bi-fundamental fields are explicitly shown on the edges.

$\mathrm{U}(1)_{\mathrm{R}}$ shall generate a one dimensional family of solutions and make a contradiction to the expected discreteness. Therefore, at least one of the edges should be removed. If there are still loops left after taking one edge to be zero, one can substitute the removed edge into the superpotential $W$ and repeat the previous argument again and again until there is no oriented loop left.

For more general cases, the $\mathrm{U}(1)_{\mathrm{R}}$ symmetry can be broken. E.g., for the quiver in figure 12 endowed with a generic superpotential,

$$
\begin{aligned}
W= & a_{i_{1} i_{4} i_{3} i_{2}} X_{i_{1}}^{(1)} X_{i_{4}}^{(4)} X_{i_{3}}^{(3)} X_{i_{2}}^{(2)}+\sum_{s=1}^{4} b_{i_{s} j_{s} k_{s}}^{(s)} X_{i_{s}}^{(s)} Y_{j_{s}}^{(s)} Z_{k_{s}}^{(s)} \\
& +c_{j_{1} k_{1} j_{2} k_{2} j_{3} k_{3} j_{4} k_{4}} Y_{j_{1}}^{(1)} Z_{k_{1}}^{(1)} Y_{j_{2}}^{(2)} Z_{k_{2}}^{(2)} Y_{j_{3}}^{(3)} Z_{k_{3}}^{(3)} Y_{j_{4}}^{(4)} Z_{k_{4}}^{(4)}
\end{aligned}
$$

one can not consistently assign the same $\mathrm{U}(1)_{\mathrm{R}}$ for every loop since there are relations among them. In such cases, we could make the following argument. Supposing that there is a unbroken loop with $N$ nodes in the quiver, let us label the corresponding nodes by $1,2, \ldots, N$, and the corresponding edges by $\vec{\Phi}^{u}, u=1, \ldots, N$. We can always construct an action of the form

$$
\begin{aligned}
\vec{\Phi}^{u} & \rightarrow \lambda_{u} \vec{\Phi}^{u}, \quad \text { for } \quad u=1, \ldots, N-1, \\
\vec{\Phi}^{N} & \rightarrow\left(\prod_{u=1}^{N-1} \lambda_{u}^{-1}\right) \vec{\Phi}^{N},
\end{aligned}
$$

to be a symmetry of the F-term equations by also assigning proper scalings

$$
\vec{\Phi}^{s \bar{t}} \rightarrow \lambda_{s \bar{t}}\left(\left\{\lambda_{u}\right\}_{u=1, \cdots, N}\right) \vec{\Phi}^{s \bar{t}}
$$

to the edges which are not in this loop. Again, it means that one can generate $N-1$ complex dimensional family of solutions and it contradicts the expected discreteness of the 

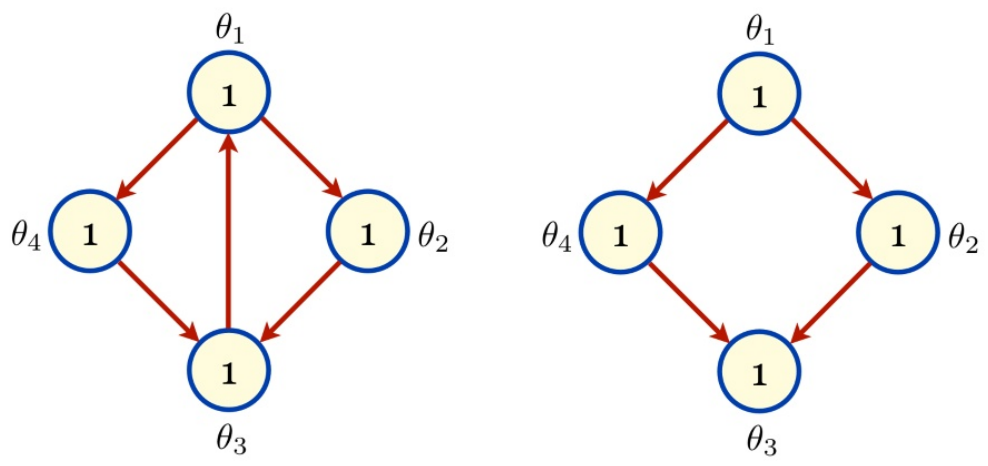

Figure 13. Abelian quiver with two loops (left), and one of its maximal reduced quivers without loop (right) obtained by eliminating the diagonal edge from node 3 to 1 ; the linking numbers are implicit.

solution. The same argument can be repeated until there is no oriented loop left. Thus, in order to have a non-zero solution in the general Abelian multi-loop case, there must be an eliminated edge in any single loop.

Given such removal of edges in a multi-loop quiver, we can derive a set of consistent conditions on the FI parameters. Unlike the cyclic case, these constraints could not fix the branch uniquely in general. That is because the reduced quiver itself could have multiple non-empty branches depend on more refined choices of FI parameters. E.g., for the fournode two-loop quiver in figure 13 (left), by removing the diagonal edge from node 3 to 1 , one can obtain the maximal reduced quiver without loop, depicted in figure 13 (right), where, by "maximal," we mean that the reduced quiver will have a loop if any one of the removed edges, which in this case is the diagonal edge, is recovered. It is easy to see that removal of this diagonal edge implies the following consistent conditions

$$
\theta_{3}>0, \quad \theta_{1}<0
$$

However, this reduced quiver may still have four different, non-trivial, branches depending on the signs of $\theta_{2}$ and $\theta_{4}$.

Thus, the branches for a Abelian multi-loop quiver are described by the non-empty branches of its maximal reduced quiver without loop. Given the maximal reduced quiver without loop and FI parameters that are read off from the original quiver, we get the D-term ambient space $X$ in the corresponding branch. The physical moduli space $M$ is decided by an intersection of the ambient space variables, and the intersecting is described by the F-term equations of the removed edges.

The main question for non-Abelian quiver is whether the F-term equations are still generic enough for the components $\Phi_{i_{s \bar{t}}}^{a_{s} \bar{b}_{t}}$ 's. By "genericity" we mean that the equations are algebraically independent. Although the coefficients of the F-term equation for $\Phi_{i_{s} \bar{t}}^{a_{s} \bar{b}_{t}}$ get repeated for the same $\left\{s, t, i_{s \bar{t}}\right\}$, they should remain algebraically independent since the variables are different for different choices of $\left\{a_{s}, \bar{b}_{t}\right\}$. Based on the fact that the F-term equations are still generic enough for the $\Phi_{i_{s} \bar{t}}^{a_{s} \bar{b}_{t}}$ s, the same argument as the Abelian case will be still valid. Thus, given an arbitrary quiver, the D-term ambient space $X$ should be 
described by the maximal reduced quiver without loop. This conclusion is consistent with the computation procedure we used in the main text.

Open Access. This article is distributed under the terms of the Creative Commons Attribution License (CC-BY 4.0), which permits any use, distribution and reproduction in any medium, provided the original author(s) and source are credited.

\section{References}

[1] F. Denef, Quantum quivers and Hall/hole halos, JHEP 10 (2002) 023 [hep-th/0206072] [INSPIRE].

[2] F. Denef and G.W. Moore, Split states, entropy enigmas, holes and halos, JHEP 11 (2011) 129 [hep-th/0702146] [INSPIRE].

[3] A. Sen, Equivalence of three wall-crossing formulae, Commun. Num. Theor. Phys. 6 (2012) 601 [arXiv: 1112.2515] [INSPIRE].

[4] H. Kim, J. Park, Z. Wang and P. Yi, Ab Initio Wall-Crossing, JHEP 09 (2011) 079 [arXiv:1107.0723] [INSPIRE].

[5] K.-M. Lee and P. Yi, Dyons in $N=4$ supersymmetric theories and three pronged strings, Phys. Rev. D 58 (1998) 066005 [hep-th/9804174] [INSPIRE].

[6] J.P. Gauntlett, N. Kim, J. Park and P. Yi, Monopole dynamics and BPS dyons $N=2$ super Yang-Mills theories, Phys. Rev. D 61 (2000) 125012 [hep-th/9912082] [INSPIRE].

[7] M. Stern and P. Yi, Counting Yang-Mills dyons with index theorems, Phys. Rev. D 62 (2000) 125006 [hep-th/0005275] [INSPIRE].

[8] F. Denef, Supergravity flows and D-brane stability, JHEP 08 (2000) 050 [hep-th/0005049] [INSPIRE].

[9] A. Ritz, M.A. Shifman, A.I. Vainshtein and M.B. Voloshin, Marginal stability and the metamorphosis of BPS states, Phys. Rev. D 63 (2001) 065018 [hep-th/0006028] [INSPIRE].

[10] P.C. Argyres and K. Narayan, String webs from field theory, JHEP 03 (2001) 047 [hep-th/0101114] [INSPIRE].

[11] S. Lee and P. Yi, Framed BPS States, Moduli Dynamics and Wall-Crossing, JHEP 04 (2011) 098 [arXiv: 1102.1729] [INSPIRE].

[12] J. Manschot, B. Pioline and A. Sen, Wall Crossing from Boltzmann Black Hole Halos, JHEP 07 (2011) 059 [arXiv: 1011.1258] [INSPIRE].

[13] J. Manschot, B. Pioline and A. Sen, A Fixed point formula for the index of multi-centered $N=2$ black holes, JHEP 05 (2011) 057 [arXiv:1103.1887] [INSPIRE].

[14] M. Kontsevich and Y. Soibelman, Stability structures, motivic Donaldson-Thomas invariants and cluster transformations, arXiv:0811.2435 [INSPIRE].

[15] D. Gaiotto, G.W. Moore and A. Neitzke, Framed BPS States, arXiv:1006.0146 [InSPIRE].

[16] S.-J. Lee, Z.-L. Wang and P. Yi, BPS States, Refined Indices and Quiver Invariants, JHEP 10 (2012) 094 [arXiv: 1207.0821] [INSPIRE].

[17] S.-J. Lee, Z.-L. Wang and P. Yi, Quiver Invariants from Intrinsic Higgs States, JHEP 07 (2012) 169 [arXiv:1205.6511] [INSPIRE]. 
[18] M. Reineke, The Harder-Narasimhan system in quantum groups and cohomology of quiver moduli, Inventiones Mathematicae 152 (2003) 349 [math/0204059].

[19] M. Alim, S. Cecotti, C. Cordova, S. Espahbodi, A. Rastogi et al., N=2 Quantum Field Theories and Their BPS Quivers, arXiv:1112.3984 [INSPIRE].

[20] A. Bertram, I. Ciocan-Fontanine and B.-s. Kim, Two proofs of a conjecture of Hori and Vafa, math/0304403 [INSPIRE].

[21] A. Bertram, I. Ciocan-Fontanine and B. Kim, Gromov-Witten Invariants for Abelian and Nonabelian Quotients, math/0407254 .

[22] I. Ciocan-Fontanine, B. Kim and C. Sabbah, The Abelian/Nonabelian correspondence and Frobenius manifolds, Inventiones Mathematicae 171 (2007) 301 [math/0610265].

[23] P. Griffiths and J. Harris, Principles of Algebraic Geometry, Wiley, 1978.

[24] S. Martin, Symplectic quotients by a nonAbelian group and by its maximal torus, Annals Math. (1999) [math/0001002] [INSPIRE].

[25] F. Hirzebruch, Topological Methods in Algebraic Geometry, Springer, 1966.

[26] L. Hille, Toric Quiver Varieties, Canadian Math. Soc. Proceedings 24 (1998) 311.

[27] H. Iritani, An integral structure in quantum cohomology and mirror symmetry for toric orbifolds, Adv. Math. 222 (2009) 1016 [arXiv:0903.1463].

[28] J. Manschot, B. Pioline and A. Sen, From Black Holes to Quivers, JHEP 11 (2012) 023 [arXiv: 1207.2230] [INSPIRE].

[29] J. Manschot, B. Pioline and A. Sen, On the Coulomb and Higgs branch formulae for multi-centered black holes and quiver invariants, JHEP 05 (2013) 166 [arXiv:1302.5498] [INSPIRE].

[30] I. Bena, M. Berkooz, J. de Boer, S. El-Showk and D. Van den Bleeken, Scaling BPS Solutions and pure-Higgs States, JHEP 11 (2012) 171 [arXiv:1205.5023] [INSPIRE].

[31] W. Fulton, Introduction to Toric Varieties, Princeton University Press, 1993.

[32] T. Oda, Convex Bodies and Algebraic Geometry, Springer-Verlag, 1988.

[33] D. Cox, Recent Developments in Toric Geometry, arXiv:alg-geom/9606016v1.

[34] D. Cox and S. Katz, Mirror Symmetry and Algebraic Geometry, American Mathematical Society, 1999.

[35] F. Denef, Les Houches Lectures on Constructing String Vacua, arXiv:0803.1194 [INSPIRE].

[36] J. Manschot, B. Pioline and A. Sen, Generalized quiver mutations and single-centered indices, JHEP 01 (2014) 050 [arXiv: 1309.7053] [INSPIRE]. 